\title{
Commutativity in the Algorithmic Lovász Local Lemma
}

\author{
Vladimir Kolmogorov \\ Institute of Science and Technology Austria \\ vnk@ist.ac .at
}

\begin{abstract}
We consider the recent formulation of the Algorithmic Lovász Local Lemma [10, 2, 3, for finding objects that avoid "bad features", or "flaws". It extends the Moser-Tardos resampling algorithm [17] to more general discrete spaces. At each step the method picks a flaw present in the current state and goes to a new state according to some prespecified probability distribution (which depends on the current state and the selected flaw). However, it is less flexible than the Moser-Tardos method since [10, 2, 3] require a specific flaw selection rule, whereas [17 allows an arbitrary rule (and thus can potentially be implemented more efficiently).

We formulate a new "commutativity" condition, and prove that it is sufficient for an arbitrary rule to work. It also enables an efficient parallelization under an additional assumption. We then show that existing resampling oracles for perfect matchings and permutations do satisfy this condition.
\end{abstract}

\section{Introduction}

Let $\Omega$ be a (large) set of objects and $F$ be a set of flaws, where a flaw $f \in F$ is some non-empty set of "bad" objects, i.e. $f \subseteq \Omega$. Flaw $f$ is said to be present in $\sigma$ if $\sigma \in f$. Let $F_{\sigma}=\{f \in F \mid \sigma \in f\}$ be the set of flaws present in $\sigma$. Object $\sigma$ is called flawless if $F_{\sigma}=\varnothing$.

The existence of flawless objects can often be shown via a probabilistic method. First, a probability measure $\omega$ on $\Omega$ is introduced, then flaws in $F$ become (bad) events that should be avoided. Proving the existence of a flawless object is now equivalent to showing that the probability of avoiding all bad events is positive. This holds if, for example, all events $f \in F$ are independent and the probability of each $f$ is smaller than 1. The well-known Lovász Local Lemma (LLL) [6] is a powerful tool that can handle a (limited) dependency between the events. Roughly speaking, it states that if the dependency graph is sparse enough (e.g. has a bounded degree) and the probabilities of individual bad events are sufficiently small then a flawless object is guaranteed to exist.

LLL has been the subject of intensive research, see e.g. [21] for a relatively recent survey. One of the milestone results was the constructive version of LLL by Moser and Tardos [17]. It applies to the variable model in which $\Omega=\mathcal{X}_{1} \times \ldots \times \mathcal{X}_{n}$ for some discrete sets $\mathcal{X}_{i}$, event $f$ depends on a small subset of variables denoted as $\operatorname{vbl}(f) \subseteq[n]$, and two events $f, g$ are declared to be dependent if $\operatorname{vbl}(f) \cap \operatorname{vbl}(g) \neq \varnothing$. The algorithm proposed in [17] is strikingly simple: (i) sample each variable $\sigma_{i}$ for $i \in[n]$ according to its distribution; (ii) while $F_{\sigma}$ is non-empty, pick an arbitrary flaw $f \in F_{\sigma}$ and resample all variables $\sigma_{i}$ for $i \in \operatorname{vbl}(f)$. Moser and Tardos proved that if the LLL condition in [6] is satisfied then the expected number of resamplings is finite (polynomial for most of the known applications).

The recent development has been extending algorithmic LLL beyond the variable model, and in particular to non-Cartesian spaces. The first such work was by Harris and Srinivasan [9], who considered the space of permutations. Achlioptas and Iliopoulos [1] introduced a more abstract 
framework where the behaviour of the algorithm is specified by a certain multigraph. Harvey and Vondrák [10] introduced the notion of resampling oracles, providing an algorithmization of the LLL given efficiently implementable resampling oracles. They also characterized the condition under which a resampling oracle exists: it was called lopsided association in [10], and was shown to lie strictly between the original asymmetric LLL condition [6] and a more refined lopsidependency LLL condition [7].

By definition, resampling oracles must satisfy a certain property intimately tying them to the LLL measure $\omega$. Achlioptas and Iliopoulos [2] gave a first analysis of LLL-inspired algorithms whose transition probabilities do not need to satisfy this property, and in [3] this analysis was simplified and extended. We will refer to this setting from [2, 3] as "general algorithmic LLL" (even though it contains cases that go beyond the probabilistic version of LLL [6, 7]).

This is the setting studied in this paper. It does not assume any particular structure on sets $\Omega$ and $F$. Instead, for each object $\sigma \in \Omega$ and flaw $f \in F_{\sigma}$ the user must provide an oracle that will used for sampling a new object. It is specified by a set of actions $A(f, \sigma) \subseteq \Omega$ that can be taken to "address" flaw $f$, and a probability distribution $\rho\left(\sigma^{\prime} \mid f, \sigma\right)$ over $\sigma^{\prime} \in A(f, \sigma)$. At each step the algorithm selects a certain flaw $f \in F_{\sigma}$, samples an action $\sigma^{\prime} \in A(f, \sigma)$ according to $\rho\left(\sigma^{\prime} \mid f, \sigma\right)$, and goes there. This framework captures the Moser-Tardos algorithm [17], and can also handle other scenarios such as permutations and perfect matchings (in which case $\Omega$ cannot be expressed as a Cartesian product).

One intriguing difference between the methods of [17] and [1, 10, 2, 3] is that [17] allows an arbitrary rule for selecting a flaw $f \in F_{\sigma}$, whereas [1, 10, 2, 3] require a specific rule (which depends on a permutation $\pi$ of $F$ chosen in advance) $)^{1}$. We will say that an algorithm is flexible if it is guaranteed to work with any flaw selection rule. We argue that flexibility can lead to a much more efficient practical implementation: it is not necessary to examine all flaws in $F_{\sigma}$, the first found flaw will suffice. If the list of current flaws is updated dynamically then flexibility could potentially eliminate the need for a costly data structure (such as a priority queue) and thus save a factor of $\Theta(\log n)$ in the complexity. The rule may also affect the number of steps in practice; experimentally, the selection process matters, as noted in [21].

Achlioptas and Iliopoulos discuss flaw selection rules in [1, Section 4.3], and remark that they do not see how to accommodate arbitrary rules in their framework. It is known, however, that in special cases flexible rules can be used even beyond the variable model. Namely, through a lengthy and a complicated analysis Harris and Srinivasan [9] managed to show the correctness of a resampling algorithm for permutations, and did not make assumptions on the flaw selection rule in their proof. They also proved a better bound for the parallel version of the algorithm.

This paper aims to understand which properties of the problem enable flexibility and parallelism. Our contributions are as follows.

- We formulate a new condition that we call "commutativity", and prove that it is sufficient for flexibility.

- We prove that it gives a better bound on the number of rounds of the parallel version of the algorithm. In particular, we show how to use commutativity for handling "partial execution logs" instead of "full execution logs" (which is required for analyzing the parallel version).

- We show that existing resampling oracles for permutations [9] and perfect matchings in complete graphs [10] are commutative. (In fact, we treat both cases in a single framework). Thus,

\footnotetext{
${ }^{1}$ The papers 1, 2 actually allowed more freedom in the choice of permutation $\pi$, e.g. it may depend on the iteration number. However, once $\pi$ has been chosen, the algorithm should still examine some "current" set of flaws and pick the lowest one with respect to $\pi$.
} 
we provide a simpler proof of the result in [9] and generalize it to other settings, in particular to perfect matchings in certain graphs (for which existing algorithms require specific rules).

To our knowledge, our commutativity condition captures all previously known cases when the flaw selection rules was allowed to be arbitrary.

Other related work Applications that involve non-Cartesian spaces $\Omega$ (such as permutations, matchings and spanning trees) have often been tackled via the Lopsided $L L L$ [7]; we refer to [15, 16] for a comprehensive survey. On the level of techniques there is some connection between this paper and a recent work by Knuth [11]; we discuss this in Section 3.

\section{Background and preliminaries}

First, we give a formal description of the general algorithmic LLL framework. As described in the previous section, we assume that for each object $\sigma \in \Omega$ and each flaw $f \in F_{\sigma}$ there is a nonempty set of actions $A(f, \sigma) \subseteq \Omega$ that can be taken for "addressing" flaw $f$ at $\sigma$, and a probability distribution $\rho\left(\sigma^{\prime} \mid f, \sigma\right)$ over $\sigma^{\prime} \in A(f, \sigma)$. Note, by definition $A(f, \sigma)$ is the support of distribution $\rho(\cdot \mid f, \sigma)$. The collection of all these distributions will be denoted as $\rho$. We fix some probability distribution $\omega$ on $\Omega$ with $\omega(\sigma)>0$ for all $\sigma \in \Omega$ (it will be used later for formulating various conditions). Note that our notation is quite different from that of Harvey and Vondrák [10]. 2] The algorithm can now be stated as follows.

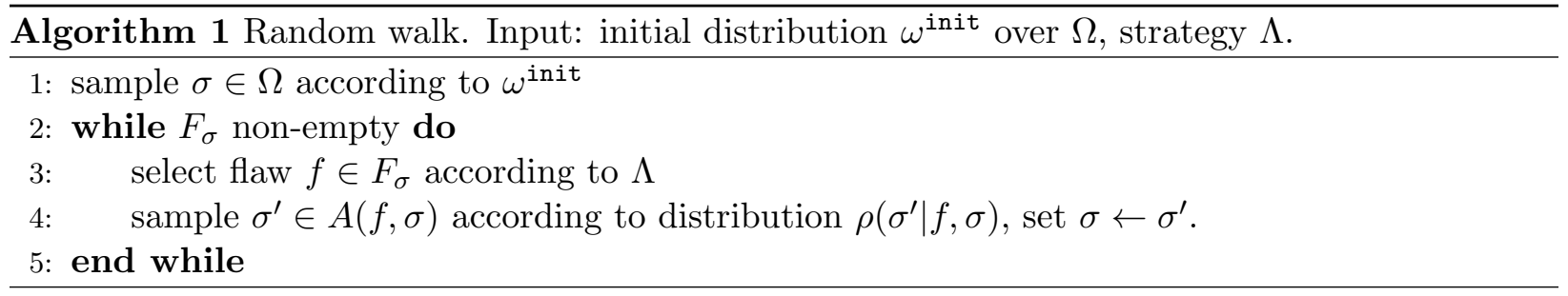

Clearly, if the algorithm terminates then it produces a flawless object $\sigma$. The works [1, 10, 2, 3] used specific strategies $\Lambda$. As stated in the introduction, our goal is to understand when an arbitrary strategy can be used. This means that flaw $f$ in line 3 is selected according to some distribution which is a function of the entire past execution history ${ }^{3}$. Note that if flaw $f \in F_{\sigma}$ in line 3 depends only on $\sigma$ then the algorithm can be viewed as a random walk in a Markov chain with states $\Omega$, while in a more general case the walk can be non-Markovian.

\subsection{Walks and the potential causality graph}

We say that $\sigma \stackrel{f}{\rightarrow} \sigma^{\prime}$ is a (valid) walk if it is possible to get from state $\sigma$ to $\sigma^{\prime}$ by "addressing" flaw $f$ as described in the algorithm, i.e. if two conditions hold: $f \in F_{\sigma}$ and $\sigma^{\prime} \in A(f, \sigma)$. Whenever we write $\sigma \stackrel{f}{\rightarrow} \sigma^{\prime}$, we mean that it is a valid walk.

In many applications sampling oracles satisfy a special condition called atomicity [1].

Definition 1. $\rho$ is called atomic if for any $f \in F$ and $\sigma^{\prime} \in \Omega$ there exists at most one object $\sigma \in \Omega$ such that $\sigma \stackrel{f}{\rightarrow} \sigma^{\prime}$.

\footnotetext{
2 "Flaws" $f$ correspond to "bad events" $E_{i}$ in $[10$. The distribution over $\Omega$ was denoted in 10 as $\mu$, the states of $\Omega$ as $\omega$, and the resampling oracle for the bad event $E_{i}$ at state $\omega \in \Omega$ as $r_{i}(\omega)$.

${ }^{3}$ The description of the algorithm in [17] says "pick an arbitrary violated event". This is consistent with our definition of an "arbitrary strategy": in the analysis Moser and Tardos mention that this selection must come from some fixed procedure (either deterministic or randomized), so that expected values are well-defined.
} 
Next, we need to describe "dependences" between flaws in $F$. Let $\sim$ be some symmetric relation on $F$ (so that $(F, \sim)$ is an undirected graph). It is assumed to be fixed throughout the paper. For a flaw $f \in F$ let $\Gamma(f)=\{g \in F \mid f \sim g\}$ be the set of neighbors of $f$. Note, we may or may not have $f \sim f$, and so $\Gamma(f)$ may or may not contain $f$. We will denote $\Gamma^{+}(f)=\Gamma(f) \cup\{f\}$, and also $\Gamma(S)=\bigcup_{f \in S} \Gamma(f)$ and $\Gamma^{+}(S)=\bigcup_{f \in S} \Gamma^{+}(f)$ for a subset $S \subseteq F$.

Definition 2. Undirected graph $(F, \sim)$ is called a potential causality graph for $\rho$ if for any walk $\sigma \stackrel{f}{\rightarrow} \sigma^{\prime}$ there holds $F_{\sigma^{\prime}} \subseteq\left(F_{\sigma}-\{f\}\right) \cup \Gamma(f)$.

In other words, $\Gamma(f)$ must contain all flaws that can appear after addressing flaw $f$ at some state. Also, $\Gamma(f)$ must contain $f$ if addressing $f$ at some state can fail to eradicate $f$.

Note that in Definition 2 we deviate slightly from [1, 2, 3]: in their analysis the potential causality graph was directed and therefore in certain cases could capture more information. While directed graphs do matter in some applications (see examples in [1, 2]), we believe that in a typical application the potential causality relation is symmetric. Using an undirected graph will be essential for incorporating commutativity.

A subset $S \subseteq F$ will be called independent if for any distinct $f, g \in S$ we have $f \nsim g$. (Thus, loops $f \sim f$ in the graph $(F, \sim)$ do not affect the definition of independence). For a subset $S \subseteq F$ we denote $\operatorname{Ind}(S)=\{T \subseteq S \mid T$ is independent $\}$.

\subsection{Commutativity}

We now formulate new conditions that will allow an arbitrary flaw selection rule to be used.

Definition 3. $(\rho, \sim)$ is called weakly commutative if there exists a mapping SWAP that sends any walk $\sigma_{1} \stackrel{f}{\rightarrow} \sigma_{2} \stackrel{g}{\rightarrow} \sigma_{3}$ with $f \nsim g$ to another valid walk $\sigma_{1} \stackrel{g}{\rightarrow} \sigma_{2}^{\prime} \stackrel{f}{\rightarrow} \sigma_{3}$, and this mapping is injective.

Note that in the atomic case the definition can be simplified. Namely, $(\rho, \sim)$ is weakly commutative if and only if it satisfies the following condition:

- For any walk $\sigma_{1} \stackrel{f}{\rightarrow} \sigma_{2} \stackrel{g}{\rightarrow} \sigma_{3}$ with $f \nsim g$ there exists state $\sigma_{2}^{\prime} \in \Omega$ such that $\sigma_{1} \stackrel{g}{\rightarrow} \sigma_{2}^{\prime} \stackrel{f}{\rightarrow} \sigma_{3}$ is also a walk.

Indeed, by atomicity the state $\sigma_{2}^{\prime}$ is unique, and so mapping SWAP in Definition 3 is constructed in a natural way. This mapping is reversible and thus injective.

For several results we will also need a stronger property.

Definition 4. $(\rho, \sim)$ is called strongly commutative if for any walk $\tau=\sigma_{1} \stackrel{f}{\rightarrow} \sigma_{2} \stackrel{g}{\rightarrow} \sigma_{3}$ with $f \nsim g$ and $\operatorname{SWAP}(\tau)=\sigma_{1} \stackrel{g}{\rightarrow} \sigma_{2}^{\prime} \stackrel{f}{\rightarrow} \sigma_{3}$ there holds

$$
\rho\left(\sigma_{2} \mid f, \sigma_{1}\right) \rho\left(\sigma_{3} \mid g, \sigma_{2}\right)=\rho\left(\sigma_{2}^{\prime} \mid g, \sigma_{1}\right) \rho\left(\sigma_{3} \mid f, \sigma_{2}^{\prime}\right)
$$

It is straightforward to check that strong commutativity holds in the variable model of Moser and Tardos. In fact, an additional property holds: for any $\sigma_{1} \stackrel{f}{\rightarrow} \sigma_{2} \stackrel{g}{\rightarrow} \sigma_{3}$ with $f \nsim g$ there exists exactly one state $\sigma_{2}^{\prime} \in \Omega$ such that $\sigma_{1} \stackrel{g}{\rightarrow} \sigma_{2}^{\prime} \stackrel{f}{\rightarrow} \sigma_{3}$. Checking strong commutativity for non-Cartesian spaces $\Omega$ is more involved; we refer to Section 6 for details. 


\subsection{Parallel version}

We will also consider the following version of the algorithm (see Algorithm 2). As presented, the algorithm is actually sequential. However, with appropriate implementations of lines 3-8 it becomes equivalent to some existing parallel algorithms, namely to the parallel algorithm of Moser and Tardos [17] in the case of the variable model, and to the parallel algorithm of Harris and Srinivasan [9] in the case of permutations. Algorithm 2 can thus be viewed as a framework for parallel algorithms. Note that it is closely related to the "MaximalSetResample" algorithm of Harvey and Vondrák [10] (see below).

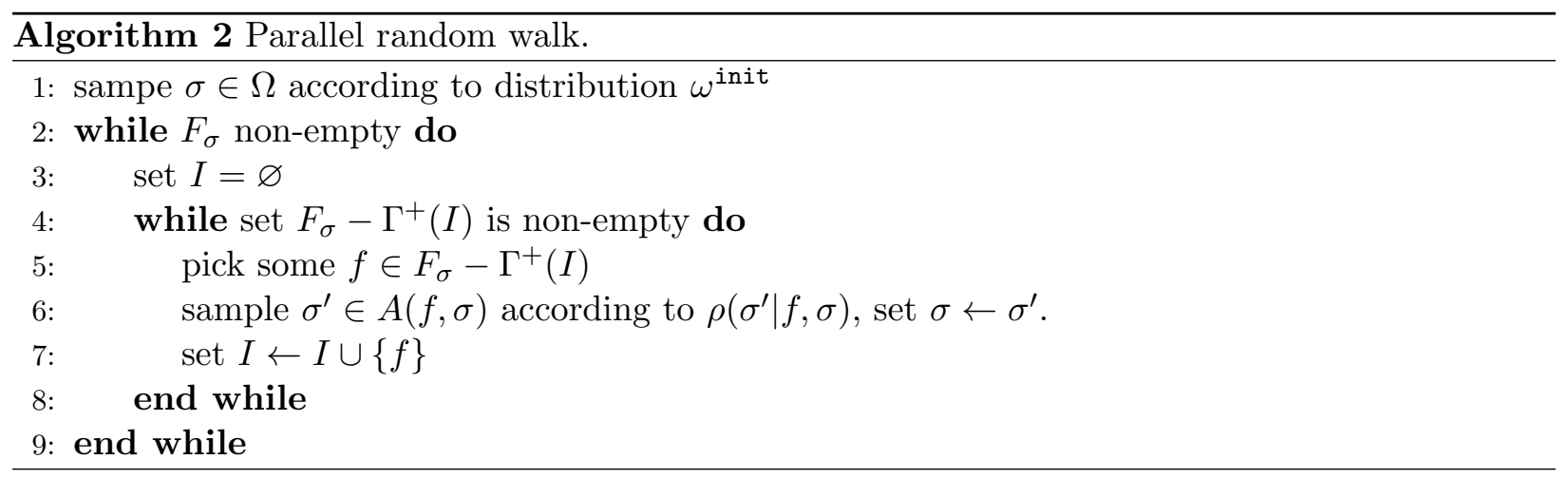

Lines 3-8 will be called a round. In some cases each round admits an efficient parallel implementation (with a polylogarithmic running time). In particular, this has been shown for the variable model of Moser and Tardos [17] and for permutations [9]. Accordingly, we will be interested in the number of rounds of the algorithm.

Note, during round $r$ the set $F_{\sigma}-\Gamma^{+}(I)$ in line 5 shrinks from iteration to iteration (and so flaw $f$ in line 5 satisfies $f \in F_{\sigma_{r}}$, where $\sigma_{r}$ is the state in the beginning of round $r$ ). This property can be easily verified using induction and Definition 2 ,

$\pi$-stable strategy Let us fix a total order $\preceq_{\pi}$ on $F$ defined by some permutation $\pi$ of $F$. Consider a version of Algorithm 2 where flaw $f$ in line 5 is selected as the lowest flaw in $F_{\sigma}-\Gamma^{+}(I)$ (with respect to $\preceq_{\pi}$ ). This corresponds to Algorithm 1 with a specific strategy $\Lambda$; this strategy will be called $\pi$-stable. It coincides with the MaximalSetResample algorithm of Harvey and Vondrák [10].

\subsection{Algorithmic LLL conditions}

In this section we formulate sufficient conditions under which a flawless object will be guaranteed to exist. The conditions involve two vectors, $\lambda$ and $\mu$. Roughly speaking, $\lambda$ characterizes sampling oracles and $\mu$ characterizes graph $(F, \sim)$.

Definition 5. The pair $(\rho, \sim)$ is said to satisfy Algorithmic LLL conditions if there exist vectors $\lambda, \mu \in \mathbb{R}^{|F|}$ such that

$$
\begin{array}{ll}
\lambda_{f} \geq \sum_{\sigma \in f} \rho\left(\sigma^{\prime} \mid f, \sigma\right) \frac{\omega(\sigma)}{\omega\left(\sigma^{\prime}\right)} & \forall f \in F, \sigma^{\prime} \in \Omega \\
\frac{\lambda_{f}}{\mu_{f}} \sum_{S \in \operatorname{Ind}(\Gamma(f))} \mu(S) \leq \theta & \forall f \in F
\end{array}
$$

where $\theta \in(0,1)$ is some constant and $\mu(S)=\prod_{g \in S} \mu_{g}$. 
Of course, vector $\lambda$ can be easily eliminated from (2). However, it is convenient to have it explicitly since in many cases it has a natural interpretation.

Note that for resampling oracles [10] one has $\lambda_{f}=\omega(f)=\sum_{\sigma \in f} \omega(\sigma)$ and an equality in (2a). The more general condition (2a) was introduced in [2, 14]; for more details we refer to [3].

Remark 1. An alternative condition that appeared in the literature (for certain $\lambda$ 's) is

$$
\frac{\lambda_{f}}{\mu_{f}} \sum_{S \subseteq \Gamma(f)} \mu(S)=\frac{\lambda_{f}}{\mu_{f}} \prod_{g \in \Gamma(f)}\left(1+\mu_{g}\right) \leq \theta
$$

Clearly, (2b) is weaker than (3). We mention that (3) is analogous to the original assymetric LLL condition in [6], while (2b) corresponds to the cluster expansion improvement by Bissacot et al. [4] (with the matching algorithmic version by Pedgen [19] who considered the variable model of Moser and Tardos). It is known that the cluster expansion version can give better results for some applications, see e.g. [5, [18, 10].

Please note a slight technical difference between the cluster expansion condition in [4] and condition (2b): in the former the sum was taken over $S \in \operatorname{Ind}\left(\Gamma^{+}(f)\right)$, while in the latter the sum is over $S \in \operatorname{Ind}(\Gamma(f))$. The latter condition can be weaker, if $\Gamma^{+}(f) \neq \Gamma(f)$ for some $f \in F$.

Shearer's condition Shearer [20] gave a sufficient and necessary condition for a general LLL to hold for a given dependency graph. Kolipaka and Szegedy [12] showed that this condition is sufficient for the Moser-Tardos algorithm, while Harvey and Vondrák [10] generalized the analysis to the framework with resampling oracles. We will show that the same analysis holds for the framework considered in this paper.

Consider vector $p \in \mathbb{R}^{|F|}$. For a subset $S \subseteq F$ denote $p^{S}=\prod_{f \in S} p_{f}$; this is a monomial in variables $\left\{p_{f} \mid f \in F\right\}$. Also, define polynomial $q_{S}$ as follows:

$$
q_{S}=q_{S}(p)=\sum_{I: S \subseteq I \in \operatorname{Ind}(F)}(-1)^{|I|-|S|} p^{I}
$$

Definition 6. Vector $p$ is said to satisfy the Shearer's condition if $q_{S}(p) \geq 0$ for all $S \subseteq F$, and $q_{\varnothing}(p)>0$.

The pair $(\rho, \sim)$ is said to satisfy Shearer's condition if there exist vector $p$ satisfying Shearer's condition, vector $\lambda$ satisfying (2a), and a constant $\theta \in(0,1)$ such that $\lambda_{f} \leq \theta \cdot p_{f}$ for all $f \in F$.

Remark 2. Bissacot et al. proved in [4] that the cluster expansion condition implies Shearer's condition. (A more explicit proof can be found in [10]). However, as mentioned in Remark 11, the condition in [4] is slightly different from (2b), so we will not be able to use this implication.

Remark 3. Note that both in condition (2b) and in the Shearer's condition we require constant $\theta$ to be strictly smaller than 1; thus, we assume that conditions hold with some slack. In the literature analogous conditions are often formulated without slack, i.e. with $\theta=1$ [20, 4, 19, 12]. The difference between slack and non-slack versions has been recently studied in detail by Harvey and Vondrák. Interestingly, if either the cluster expansion condition or the Shearer's condition holds with $\theta=1$ then it also holds with $\theta<1$, because conditions define open regions [10].

We do not know whether the same is true in the case of condition (2b) (since it is slightly different from the cluster expansion condition). 


\section{Our results}

First, we state our results for the sequential version (Algorithm 1). Unless mentioned otherwise, the flaw selection strategy and the initial distribution $\omega^{\text {init }}$ are assumed to be arbitrary.

Theorem 7. Suppose that $(\rho, \sim)$ satisfies either condition (2) or Shearer's condition, and one of the following holds:

(a) $(\rho, \sim)$ is weakly commutative and atomic.

(b) $(\rho, \sim)$ is strongly commutative.

Define

$$
\gamma^{\text {init }}=\max _{\sigma \in \Omega} \frac{\omega^{\text {init }}(\sigma)}{\omega(\sigma)}, \quad \quad \operatorname{Ind}{ }^{\text {init }}= \begin{cases}\bigcup_{\sigma \in \operatorname{supp}\left(\omega^{\text {init }}\right)} \operatorname{Ind}\left(F_{\sigma}\right) & \text { in the case }(a) \\ \operatorname{Ind}(F) & \text { in the case }(b)\end{cases}
$$

where $\operatorname{supp}\left(\omega^{\text {init }}\right)=\left\{\sigma \in \Omega \mid \omega^{\text {init }}(\sigma)>0\right\}$ is the support of $\omega^{\text {init }}$. The probability that Algorithm 1 produces a flawless object in fewer than $T+r$ steps is at least $1-\theta^{r}$ where

$$
T=\frac{1}{\log \theta^{-1}}\left(\log \gamma^{\text {init }}+\log \sum_{R \in \text { Ind }^{\text {init }}} \mu(R)\right)
$$

and $\mu(R)=\prod_{f \in R} \mu_{f}$ (in the case of condition (2)) or $\mu(R)=\frac{q_{R}(p)}{q_{\varnothing}(p)}$ (in the case of the Shearer's condition).

Next, we analyze the parallel version.

Theorem 8. Suppose that $(\rho, \sim)$ satisfies either condition (2) or the Shearer's condition, and is strongly commutative. Then the probability that Algorithm 2 produces a flawless object in fewer than $T+r$ rounds is at least $1-\theta^{r}$ where

$$
T=\frac{1}{\log \theta^{-1}}\left(\log \gamma^{\text {init }}+\log \sum_{f \in F} \mu(\{f\})\right)
$$

where $\gamma^{\text {init }}$ and $\mu(\{f\})$ are as defined in Theorem 7 . In particular, $\mu(\{f\})=\mu_{f}$ in the case of condition (2) and $\mu(\{f\})=\frac{q_{\{f\}}(p)}{q_{\varnothing}(p)}$ in the Shearer's case.

Remark 4. The possibility of using distribution $\omega^{\text {init }}$ which is different from $\omega$ was first proposed by Achlioptas and Iliopoulos in [1]. Namely, they used a distribution with $\left|\operatorname{supp}\left(\omega^{\text {init }}\right)\right|=1$, and later extended it to arbitrary distributions $\omega^{\text {init }}$ in [2]. There is a trade-off in choosing $\omega^{\text {init }}$ : smaller $\operatorname{supp}\left(\omega^{\text {init }}\right)$ leads to a smaller set Ind $^{\text {init }}$ but increases the constant $\gamma^{\text {init }}$. It is argued in [2] that using $\omega^{\text {init }} \neq \omega$ can be beneficial when sampling from $\omega$ is a difficult problem, or when the number of flaws is exponentially large.

The techniques we develop to deal with the commutative case, yielding Theorems 7 and 8 above, also give a new result for the non-commutative setting.

Theorem 9. Suppose that Algorithm 1 uses a $\pi$-stable strategy and $(\rho, \sim)$ satisfies either condition (2) or the Shearer's condition. Then, the same conclusions hold as for case (a) of Theorem 7 . 
We note that while Theorem 7 was already largely established in earlier works [10, 2, 3], the combination of condition (2a) with the Shearer's condition is new. We include it mainly because its proof serves to introduce several notions from earlier works needed to prove our main results concerning the commutative setting.

Our techniques The general idea of the proofs is to construct a "swapping mapping" that transforms "walks" (which are possible executions of the algorithm) to some canonical form by applying swap operations from Definition 3. Importantly, we need to make sure that the mapping is injective: this will guarantee that the sum over original walks is smaller or equal than the sum over "canonical walks". We then upper-bound the latter sum using some standard techniques [12, 10]. We use two approaches:

1. Theorem 7(a): transforming walks to "forward stable sequences" (a forward-looking analysis). This works only in the atomic case (under the weak commutativity assumption), and can make use of the knowledge of the set $\operatorname{supp}\left(\omega^{\text {init }}\right)$ leading to a tighter definition of the set Ind $^{\text {init }}$.

2. Theorems 7(b) and 8 transforming walks to "backward stable sequences" (a backward-looking analysis). This works in the non-atomic cases, but requires strong commutativity. In this approach the "roots" of stable sequences are on the right, and have no connection to $\omega^{\text {init }}$; this means that we must use $\operatorname{Ind}^{\text {init }}=\operatorname{Ind}(F)$.

Analyzing the parallel version requires dealing with "partial execution logs" instead of "full execution logs". It appears that this is possible only with backward sequences.

Note that previously a backward-looking analysis (with either "stable sequences" or "witness trees") was used for the variable model of Moser and Tardos [17, 12, 19] and for permutations [9], while a forward-looking analysis was used for LLL versions on non-Cartesian spaces [1, 10, 2] and also on Cartesian spaces [8].

After the first version of this work [13] we learned about a recent book draft by Knuth [11]. He considers the variable model of Moser-Tardos, and gives an alternative proof of algorithm's running time which is also based on swapping arguments (justified by a technique of "coupling" two random sources, similar to [17]). We emphasize that we go beyond the variable model, in which case justifying "swapping" seems to require different techniques.

The proofs of Theorems 7 and 8 are given below in Sections 4 and 5 . (A brief overview of these proofs can be found in [14]). The most technical part is probably constructing an injective swapping mapping for transforming to backward stable sequences (proved in Section 5.5). In Section 6 we describe our third result, which is a proof of strong commutativity of some existing resampling oracles. We also consider one application, namely rainbow matchings in complete graphs.

\section{Proof of Theorem 9}

Before jumping to the commutative case, we will need to give a background on the non-commutative case. Accordingly, in this section we recall a proof of Theorem 9. As mentioned in Section 3, various versions of this theorem have been established earlier, and techniques used in this section are a combination of ideas from previous works [12, 1, 10, 2, 3.

We write $f \cong g$ for flaws $f, g \in F$ if either $f \sim g$ or $f=g$ (and $f \not \approx g$ otherwise).

A walk of length $t$ is a sequence $\tau=\sigma_{1} \stackrel{w_{1}}{\rightarrow} \sigma_{2} \ldots \sigma_{t} \stackrel{w_{t}}{\rightarrow} \sigma_{t+1}$ such that $w_{i} \in F_{\sigma_{i}}$ and $\sigma_{i+1} \in$ 
$A\left(w_{i}, \sigma_{i}\right)$ for $i \in[t]$. Its length is denoted as $|\tau|=t$. For such a walk we define quantity

$$
p(\tau)=\omega^{\text {init }}\left(\sigma_{1}\right) \cdot \prod_{i=1}^{t} \rho\left(\sigma_{i+1} \mid w_{i}, \sigma_{i}\right)
$$

Let $\Lambda$ be the strategy for selecting flaws used in Algorithm 1. We assume in the analysis that this strategy is deterministic, i.e. the flaw $w_{i}$ in a walk $\tau=\sigma_{1} \stackrel{w_{1}}{\rightarrow} \ldots \stackrel{w_{i-1}}{\rightarrow} \sigma_{i} \stackrel{w_{i}}{\rightarrow} \ldots$ is uniquely determined by the previous history $\tau_{i}=\sigma_{1} \stackrel{w_{1}}{\rightarrow} \ldots \stackrel{w_{i-1}}{\rightarrow} \sigma_{i}$. This assumption can be made w.l.o.g.: if $\Lambda$ is randomized (i.e. a distribution over some set of deterministic strategies) then the claim of Theorem 7 can be obtained by taking the appropriate expectation over strategies (whose number is finite for a fixed finite $t$ ). A similar argument applies to Theorem 8 .

A walk $\tau$ of length $t$ that can be produced by Algorithm 1 with a positive probability will be called a bad t-trajectory. Equivalently, it is a walk that starts at a state $\sigma \in \operatorname{supp}\left(\omega^{\text {init }}\right)$ and follows strategy $\Lambda$. Note that it goes only through flawed states (except possibly the last state). Let $\operatorname{Bad}(t)$ be the set of all bad $t$-trajectories. Clearly, for any $\tau \in \operatorname{Bad}(t)$ the probability that the algorithm will produce $\tau$ equals $p(\tau)$, as defined in (7). This gives

Proposition 10. The probability that Algorithm 1 takes $t$ steps or more equals $\sum_{\tau \in \operatorname{Bad}(t)} p(\tau)$.

If $W=w_{1} \ldots w_{t}$ is the complete sequence of flaws in a walk $\tau$ then we will write $\tau \doteq W$. If we want to indicate certain intermediate states of $\tau$ then we will write them in square brackets at appropriate positions, e.g. $\tau \stackrel{\bullet}{\doteq}\left[\sigma_{1}\right] w_{1} w_{2}\left[\sigma_{3}\right] w_{4} w_{5}\left[\sigma_{6}\right]$.

In general, a sequence of flaws will be called a word, and a sequence of flaws together with some intermediate states (such as $\left[\sigma_{1}\right] w_{1} w_{2}\left[\sigma_{3}\right] w_{4} w_{5}\left[\sigma_{6}\right]$ ) will be called a pattern. For a pattern $X$ we define $\langle X\rangle=\{\tau \mid \tau \stackrel{\bullet}{=}\}$ to be the set of walks consistent with $X$. The number of flaws in $X$ is denoted as $|X|$. The following has been shown in [3] using an induction argument.

Lemma 11 ([3]). For any word $W$ and state $\sigma$ we have

$$
\begin{aligned}
\sum_{\tau \in\langle W[\sigma]\rangle} p(\tau) & \leq \gamma^{\text {init }} \cdot \lambda_{W} \cdot \omega(\sigma) \\
\sum_{\tau \in\langle W\rangle} p(\tau) & \leq \gamma^{\text {init }} \cdot \lambda_{W}
\end{aligned}
$$

where for a word $W=w_{1} \ldots w_{t}$ we denoted $\lambda_{W}=\prod_{i=1}^{t} \lambda_{w_{i}}$. (As described in the previous paragraph, $\langle W[\sigma]\rangle$ is the set of walks $\tau$ whose sequence of flaws is $W$ and the last state is $\sigma$.

The following technical result will also be useful.

Lemma 12. Consider a walk $\tau \doteq \ldots[\sigma] u_{1} \ldots u_{k} \ldots$ where $k \geq 1$. Suppose at least one of the following holds:

(a) $u_{k}$ is not present in $\sigma$.

(b) $u_{1}=u_{k}$.

Then there exists index $i \in[k-1]$ such that $u_{i} \sim u_{k}$.

Proof. We will assume that $\tau=\ldots \sigma_{1} \stackrel{u_{1}}{\rightarrow} \sigma_{2} \stackrel{u_{2}}{\rightarrow} \ldots \stackrel{u_{k-1}}{\rightarrow} \sigma_{k} \stackrel{u_{k}}{\rightarrow} \sigma_{k+1} \ldots$ where $\sigma_{1}=\sigma$.

(a) Flaw $u_{k}$ is present in $\sigma_{k}$ (since $\sigma_{k} \stackrel{u_{k}}{\rightarrow} \sigma_{k+1}$ is a walk) but not in $\sigma_{1}$. Thus, there must exist index $i \in[k-1]$ such that $u_{k}$ is present in $\sigma_{i+1}$ but not in $\sigma_{i}$. We know that $\sigma_{i} \stackrel{u_{i}}{\rightarrow} \sigma_{i+1}$ is a valid walk. Thus, addressing $u_{i}$ has caused $u_{k}$ to appear, and therefore $u_{i} \sim u_{k}$. 
(b) Assume that $u_{k}$ is present in $\sigma_{1}$ (otherwise the claim holds by (a)). If $u_{k}$ is present in $\sigma_{2}$ then $u_{k} \sim u_{k}$ (since addressing $u_{1}=u_{k}$ at state $\sigma_{1}$ did not eliminate flaw $u_{k}$ ). Otherwise, if $u_{k}$ is not present in $\sigma_{2}$, we can apply part (a) and conclude that $u_{i} \sim u_{k}$ for some $i \in[2, k-1]$.

\subsection{Stable walks and stable sequences}

As shown in [10, if $\Lambda$ is a $\pi$-stable strategy then walks $\tau$ that it produces have a special structure: the word $W$ corresponding to $\tau$ can be uniquely described by a stable sequence. This section gives all necessary definitions.

Definition 13. A sequence of sets $\varphi=\left(I_{1}, \ldots, I_{s}\right)$ with $s \geq 1$ is called stable if $I_{r} \in \operatorname{Ind}(F)$ for each $r \in[s]$ and $I_{r+1} \subseteq \Gamma^{+}\left(I_{r}\right)$ for each $r \in[s-1]$.

Definition 14. A word $W=w_{1} \ldots w_{t}$ is called stable if it can be partitioned into non-empty words as $W=W_{1} \ldots W_{s}$ such that flaws in each word $W_{r}$ are distinct, and the sequence $\left(I_{1}, \ldots, I_{s}\right)$ is stable where $I_{r}$ is the set of flaws in $W_{r}$ (for $r \in[s]$ ). If in addition each word $W_{r}=w_{i} \ldots w_{j}$ satisfies $w_{i} \prec_{\pi} \ldots \prec_{\pi} w_{j}$ then $W$ is called $\pi$-stable.

A walk $\tau \doteq W$ is called stable ( $\pi$-stable) if the word $W$ is stable ( $\pi$-stable).

It can be seen that for a stable word the partitioning in Definition 14 is unique, and can be obtained by the following algorithm. Start with one segment containing $w_{1}$, and then for $i=2, \ldots, t$ do the following: if there exists flaw $w_{k}$ in the last segment with $w_{k} \cong w_{i}$ then start a new segment containing $w_{i}$, otherwise add $w_{i}$ to the last segment. (If this algorithm is applied to an arbitrary word $W$ then it may fail to produce a stable sequence since in the latter case, when $w_{i}$ is added to the last segment $I_{r}, w_{i}$ may not belong to $\Gamma^{+}\left(I_{r-1}\right)$ ).

For a stable word $W$ let $R_{W}$ be the first set (the "root") of the stable sequence $\varphi=\left(I_{1}, \ldots, I_{s}\right)$ corresponding to $W$, i.e. $R_{W}=I_{1}$. (If $W$ is empty then $R_{W}=\varnothing$ ). Let $\mathrm{Stab}_{\pi}$ be the set of $\pi$-stable words $W$ for which there exists a walk $\tau$ with $\tau \stackrel{\bullet}{\doteq}$. Denote

$$
\begin{aligned}
\operatorname{Stab}_{\pi}(R) & =\left\{W \in \operatorname{Stab}_{\pi}: R_{W}=R\right\} \\
\operatorname{Stab}_{\pi}(R, t) & =\left\{W \in \operatorname{Stab}_{\pi}(R):|W| \geq t\right\}
\end{aligned}
$$

The following result is proven in Section A using techniques from [12, 10].

Theorem 15. Suppose that $(\rho, \sim)$ satisfies either the cluster expansion condition (2b) or the Shearer's condition from Definition 6 . Then

$$
\sum_{W \in \operatorname{Stab}_{\pi}(R, t)} \lambda_{W} \leq \mu(R) \cdot \theta^{t} \quad \forall R \in \operatorname{Ind}(F)
$$

Recall that $\mu(R)=\prod_{f \in R} \mu_{f}$ in the case of condition (2) and $\mu(R)=\frac{q_{R}(p)}{q_{\varnothing}(p)}$ in the Shearer's case.

For some parts of the proofs we will need to use the reverse of stable walks and sequences. (This will correspond to a "backward-looking analysis" instead of the "forward-looking analysis"). The necessarly definitions are given below.

A sequence $\phi=\left(I_{1}, \ldots, I_{s}\right)$ will be called reversely stable if its reverse $\left(I_{s}, \ldots, I_{1}\right)$ is a stable sequence. A word $W=w_{1} \ldots w_{t}$ will be called reversely stable (reversely $\pi$-stable) if its reverse $w_{t} \ldots w_{1}$ is stable $(\pi$-stable). A reversely stable and reversely $\pi$-stable walks $\tau$ are defined in an analogous way. 
For a reversely stable word $W$ let $R_{W}^{\text {rev }}$ be the last set of the reversely stable sequence $\phi=$ $\left(I_{1}, \ldots, I_{s}\right)$ corresponding to $W$, i.e. $R_{W}^{\text {rev }}=I_{s}$. Let $\mathrm{Stab}_{\pi}^{\text {rev }}$ be the set of reversely $\pi$-stable words $W$ for which there exists a walk $\tau$ with $\tau \doteq W$. For $R \subseteq F$, denote

$$
\begin{aligned}
\operatorname{Stab}_{\pi}^{\text {rev }}(R) & =\left\{W \in \mathrm{Stab}_{\pi}^{\text {rev }}: R_{W}^{\text {rev }}=R\right\} \\
\operatorname{Stab}_{\pi}^{\text {rev }}(R, t) & =\left\{W \in \operatorname{Stab}_{\pi}^{\text {rev }}(R):|W| \geq t\right\}
\end{aligned}
$$

Theorem 16. Suppose that $(\rho, \sim)$ satisfies either the cluster expansion condition (2b) or the Shearer's condition from Definition [6. Then

$$
\sum_{W \in \operatorname{Stab}_{\pi}^{\text {rev }}(R, t)} \lambda_{W} \leq \mu(R) \cdot \theta^{t} \quad \forall R \in \operatorname{Ind}(F)
$$

Note that set $\mathrm{Stab}_{\pi}^{\text {rev }}$ does not necessarily equal the reverse of words from $\mathrm{Stab}_{\pi}$ (because of the condition "there exists walk $\tau$ with $\tau \doteq W$ " present in the definitions of both $\mathrm{Stab}_{\pi}$ and $\mathrm{Stab}_{\pi}^{\text {rev }}$ ). Thus, Theorem 16 does not automatically follow from Theorem 15 . Their proofs, however, are very similar (see Section A).

\subsection{Proof of Theorem 9; a wrap-up}

It is not difficult to show that a $\pi$-stable strategy produces $\pi$-stable walks (see Proposition 17 below). The reverse, however, is not necessary true: it may e.g. happen that some flaw $f$ does not appear in a $\pi$-stable walk $\tau$, but is present in all states of $\tau$ and would have been selected by any $\pi$-stable strategy.

Proposition 17. Suppose that strategy $\Lambda$ is implemented as in Algorithm 2 (with a deterministic choice in line 5). Then any $\tau \in \operatorname{Bad}(t)$ is a stable walk. If in addition $\Lambda$ is a $\pi$-stable strategy (i.e. flaw $f$ in line 5 is chosen as the lowest flaw in $F_{\sigma}-\Gamma^{+}(I)$ with respect to $\left.{ }_{\pi}\right)$ then any $\tau \in \operatorname{Bad}(t)$ is a $\pi$-stable walk.

Proof. Let $s$ be the number of rounds of Algorithm 2 that produced walk $\tau$, and $I_{r} \subseteq F$ be the set of flaws addressed in round $r \in[s]$, or equivalently the set $I$ at the end of round $r$ (with a possible exception for $r=s$ : $I_{r}$ may correspond to the "intermediate" set $I$, depending on where walk $\tau$ was "cut"). By this definition, we have $\tau \stackrel{\bullet}{=} W_{1} \ldots W_{s}$ where $W_{r}$ is a word containing the flaws in $I_{r}$ in some order (with $\left|W_{r}\right|=\left|I_{r}\right|$ ). We will prove that $\left(I_{1}, \ldots, I_{s}\right.$ ) is a stable sequence; this will imply the first claim of the proposition.

The independence of each set $I_{r}$ follows directly from the construction. Consider $r \in[2, s]$, and let $\tau \doteq \ldots W_{r-1}[\sigma] W_{r} \ldots$. We need to show that for each flaw $f$ present in $W_{r}$ (i.e. $f \in I_{r}$ ) we have $f \in \Gamma^{+}\left(I_{r-1}\right)$. Suppose it is not the case. Lemma 12(a) gives that $f$ is present in $\sigma$. Therefore, set $F_{\sigma}-\Gamma^{+}\left(I_{r-1}\right)$ is non-empty (it contains $\sigma$ ). But then round $r-1$ would not have terminated at the state $\sigma$ - a contradiction.

It remains to consider the case when flaw $f$ in line 5 is chosen as the lowest flaw in $F_{\sigma}-\Gamma^{+}(I)$ with respect to $\preceq_{\pi}$. Consider round $r$, and let $W_{r}=w_{i} \ldots w_{j}$. Definition 2 and an induction argument show that during this round set $F_{\sigma}-\Gamma^{+}(I)$ in line 5 shrinks from iteration to iteration. This implies that $w_{i} \prec_{\pi} \ldots \prec_{\pi} w_{j}$, as desired.

We also need the following observation.

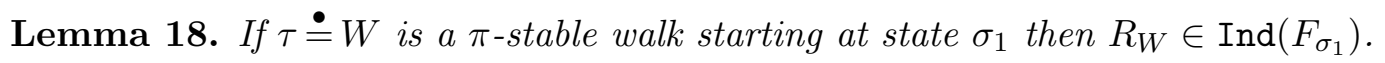


Proof. By definition of a stable word, set $R_{W}$ is independent, and corresponds to some prefix $w_{1} \ldots w_{k}$ of the word $W$. It remains to show that for each $i \in[k]$ we have $w_{i} \in F_{\sigma_{1}}$. This follows from Lemma 12(a) and the condition that $w_{j} \nsim w_{i}$ for all $j \in[i-1]$.

We now have all ingredients to prove Theorem 9. Consider Algorithm 1 with a $\pi$-stable strategy. Then each walk $\tau \doteq W$ from $\operatorname{Bad}(t)$ is $\pi$-stable, with $W \in \operatorname{Stab}_{\pi}(t)$. By the definition of $\operatorname{Bad}(t)$ and Lemma 18 we also know that $R_{W} \in \operatorname{Ind}^{\text {init }}=\bigcup_{\sigma \in \operatorname{supp}\left(\omega^{\text {init }}\right)} \operatorname{Ind}\left(F_{\sigma}\right)$. Therefore,

$$
\begin{aligned}
\operatorname{Pr}[\# \text { steps } \geq t] & =\sum_{\tau \in \operatorname{Bad}(t)} p(\tau) \leq \sum_{R \in \operatorname{Ind}^{\text {init }}} \sum_{W \in \operatorname{Stab}_{\pi}(R, t)} \sum_{\tau \in\langle W\rangle} p(\tau) \\
& \stackrel{(\text { a) }}{\leq} \sum_{R \in \operatorname{Ind}^{\text {init }}} \sum_{W \in \operatorname{Stab}_{\pi}(R, t)} \gamma^{\text {init }} \cdot \lambda_{W} \stackrel{\text { (b) }}{\leq} \gamma^{\text {init }} \cdot \sum_{R \in \operatorname{Ind}^{\text {init }}} \mu(R) \cdot \theta^{t}=\theta^{t-T}
\end{aligned}
$$

where (a) holds by Lemma 11, (b) holds by Theorem 15, and $T$ is given by the expression in (5):

$$
T=\frac{1}{\log \theta^{-1}}\left(\log \gamma^{\text {init }}+\log \sum_{R \in \text { Ind }^{\text {init }}} \mu(R)\right)
$$

\section{Commutativity and swapping mappings}

From now on we assume that $(\rho, \sim)$ is weakly commutative. Therefore, for any walk $\tau=\ldots \sigma_{1} \stackrel{f}{\rightarrow} \sigma_{2} \stackrel{g}{\rightarrow} \sigma_{3} \ldots$ with $f \neq g$ there exists another walk $\tau^{\prime}=\ldots \sigma_{1} \stackrel{g}{\rightarrow} \sigma_{2}^{\prime} \stackrel{f}{\rightarrow} \sigma_{3} \ldots$ obtained from $\tau$ by applying the SWAP operator to the subwalk $\sigma_{1} \stackrel{f}{\rightarrow} \sigma_{2} \stackrel{g}{\rightarrow} \sigma_{3}$. Such operation will be called a valid swap applied to $\tau$. A mapping $\Phi$ on a set of walks that works by applying some sequence of valid swaps will be called a swapping mapping. Note that if $\tau^{\prime}=\Phi(\tau)$ then the first and the last states of $\tau^{\prime}$ coincide with that

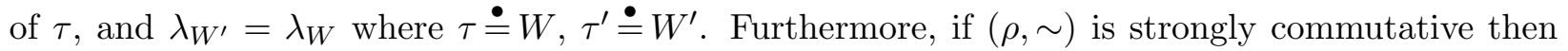
$p\left(\tau^{\prime}\right)=p(\tau)$.

We now deal with the case when $\Lambda$ is an arbitrary deterministic strategy, and so walks $\tau \in \operatorname{Bad}(t)$ are not necessarily $\pi$-stable. Our approach will be to construct a bijective swapping mapping $\Phi$ that sends walks $\tau \in \operatorname{Bad}(t)$ to some canonical walks, namely either to $\pi$-stable walks (which will work only in the atomic case) or to reversely $\pi$-stable walks (which will work in the general case). Theorems 20(a), 20(b) and 21 below give three ways to construct such mappings; they will be used for the proofs of Theorems $7(\mathrm{a}), 7(\mathrm{~b})$ and 8 , respectively.

We will need a few definitions first. A generalized walk is a formal finite sequence $\tau=$ $\left[\sigma_{1}\right] w_{1}\left[\sigma_{2}\right] w_{2} \ldots$ with $w_{i} \in F_{\sigma_{i}}$ and $\sigma_{i+1} \in A\left(w_{i}, \sigma_{i}\right)$ for all $i$. Note that $\tau$ can either end with a state $\left(\tau=\ldots\left[\sigma_{t}\right]\right)$, or end with a flaw $\left(\tau=\ldots\left[\sigma_{t}\right] w_{t}\right)$, or be empty $(\tau=\epsilon)$. In the first case $\tau$ is a usual walk. To indicate this case, we will write $\tau=\ldots[\Omega]$. We emphasize that by a "walk" we always mean a sequence of the form $\tau=\ldots[\Omega]$, unless we explicitly use the word "generalized". For two generalized walks $\tau, \tau^{\prime}$ their largest common prefix is denoted as $\tau \wedge \tau^{\prime}$ (it is itself a generalized walk).

Definition 19. A set of walks $\mathcal{X}$ is called valid if $\tau \wedge \tau^{\prime} \neq \ldots[\Omega]$ for all distinct $\tau, \tau^{\prime} \in \mathcal{X}$.

It can be seen that $\mathcal{X}$ is valid if two conditions hold: (i) there exists a deterministic strategy $\tilde{\Lambda}$ in Algorithm 1 such that all walks in $\mathcal{X}$ follow $\tilde{\Lambda}$, and (ii) for any $\tau, \tau^{\prime} \in \mathcal{X}$, walk $\tau$ is not a proper prefix of $\tau^{\prime}$. In particular, set $\operatorname{Bad}(t)$ is valid. 
Theorem 20. Suppose that $(\rho, \sim)$ is weakly commutative, and $\mathcal{X}$ is a valid set of walks.

(a) Suppose in addition that $(\rho, \sim)$ is atomic. Then there exists a set of $\pi$-stable walks $\mathcal{X}_{\pi}$ and $a$ swapping mapping $\Phi: \mathcal{X} \rightarrow \mathcal{X}_{\pi}$ which is a bijection.

(b) In a general case there exists a set of reversely $\pi$-stable walks $\mathcal{X}_{\pi}$ and a swapping mapping $\Phi: \mathcal{X} \rightarrow \mathcal{X}_{\pi}$ which is a bijection.

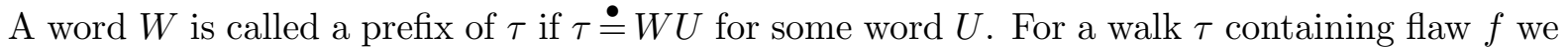
define word $\operatorname{PREFIX}^{f}(\tau)$ as the longest prefix of $\tau$ that ends with $f$. Thus, we have $\tau \doteq \operatorname{PREFIX}^{f}(\tau) U$ where $\operatorname{PREFIX} f(\tau)=\ldots f$ and word $U$ does not contain $f$.

Theorem 21. Suppose that $(\rho, \sim)$ weakly commutative, and $\mathcal{X}$ is a valid set of walks containing some fixed flaw $f \in F$. There exists a set of walks $\mathcal{X}_{\pi}$ and a swapping mapping $\Phi: \mathcal{X} \rightarrow \mathcal{X}_{\pi}$ which is a bijection such that

(i) for any $\tau \in \mathcal{X}_{\pi}$ the word $W=\operatorname{PREFIX} f(\tau)$ is reversely $\pi$-stable with $R_{W}^{\mathrm{rev}}=\{f\}$;

(ii) for any word $W$ the set $\left\{\tau \in \mathcal{X}_{\pi} \mid \operatorname{PREFIX}{ }^{f}(\tau)=W\right\}$ is valid.

We prove Theorems 20,21 in Sections 5.4 and 5.5, but first we show how they imply Theorems 7 and 8

\subsection{Proof of Theorem 7(a) (sequential algorithm in the atomic case)}

The assumption that $(\rho, \sim)$ is atomic gives the following observation.

Proposition 22 ([1]). Walk $\tau=\sigma_{1} \stackrel{w_{1}}{\rightarrow} \sigma_{2} \ldots \sigma_{t} \stackrel{w_{t}}{\rightarrow} \sigma_{t+1}$ can be uniquely reconstructed from the sequence of flaws $w_{1} \ldots w_{t}$ and the final state $\sigma_{t+1}$.

Proof. By atomicity, state $\sigma_{i}$ can be uniquely reconstructed from the flaw $w_{i}$ and the state $\sigma_{i+1}$. Applying this argument for $i=t, t-1, \ldots, 1$ gives the claim.

The proposition allows us to write walks more compactly as $\tau=w_{1} \ldots w_{t}\left[\sigma_{t+1}\right]$. Also, Lemma 11 gives for a walk $\tau=W\left[\sigma_{t+1}\right]$ that

$$
p(\tau) \leq \gamma^{\text {init }} \cdot \lambda_{W} \cdot \omega\left(\sigma_{t+1}\right)
$$

Let us apply Theorem 20(a) with $\mathcal{X}=\operatorname{Bad}(t)$. Denoting $\operatorname{Bad}_{\pi}(t)=\mathcal{X}_{\pi}$, we can write

$$
\begin{aligned}
\operatorname{Pr}[\# \text { steps } \geq t] & =\sum_{\tau \in \operatorname{Bad}(t)} p(\tau) \stackrel{(\text { a) }}{\leq} \sum_{\tau=W[\sigma] \in \operatorname{Bad}(t)} \gamma^{\text {init }} \cdot \lambda_{W} \cdot \omega(\sigma) \\
& \stackrel{(\text { b) }}{=} \sum_{\tau=W[\sigma] \in \operatorname{Bad}_{\pi}(t)} \gamma^{\text {init }} \cdot \lambda_{W} \cdot \omega(\sigma) \\
& \leq \gamma^{\text {init }} \cdot \sum_{R \in \operatorname{Ind}^{\text {init }}} \sum_{W \in \operatorname{Stab}_{\pi}(R, t)} \sum_{\sigma \in \Omega} \lambda_{W} \cdot \omega(\sigma) \\
& \leq \gamma^{\text {init }} \cdot \sum_{R \in \operatorname{Ind}^{\text {init }}} \sum_{W \in \operatorname{Stab}_{\pi}(R, t)} \lambda_{W} \leq \gamma^{\text {init }} \cdot \sum_{R \in \operatorname{Ind}^{\text {init }}} \mu(R) \cdot \theta^{t}=\theta^{t-T}
\end{aligned}
$$

where in (a) we used eq. (11), in (b) we use bijectiveness of mapping $\Phi$, and the rest is similar to the derivation in Section 4.2 . 


\subsection{Proof of Theorem 7 (b) (sequential algorithm with strong commutativity)}

In this case we have Ind ${ }^{\text {init }}=\operatorname{Ind}(F)$. Let us apply Theorem 20(b) with $\mathcal{X}=\operatorname{Bad}(t)$. Denoting $\operatorname{Bad}_{\pi}(t)=\mathcal{X}_{\pi}$, we can write

$$
\begin{aligned}
\operatorname{Pr}[\# \text { steps } \geq t] & =\sum_{\tau \in \operatorname{Bad}(t)} p(\tau) \stackrel{(\text { a) }}{=} \sum_{\tau \in \operatorname{Bad}_{\pi}(t)} p(\tau) \leq \sum_{R \in \operatorname{Ind}(F)} \sum_{W \in \operatorname{Stab}_{\pi}^{\text {rev }}(R, t)} \sum_{\tau \in\langle W\rangle} p(\tau) \\
& \leq \sum_{R \in \operatorname{Ind}(F)} \sum_{W \in \operatorname{Stab}_{\pi}^{\text {rev }}(R, t)} \gamma^{\text {init }} \cdot \lambda_{W} \leq \gamma^{\text {init }} \cdot \sum_{R \in \operatorname{Ind}(F)} \mu(R) \cdot \theta^{t}=\theta^{t-T}
\end{aligned}
$$

where in (a) we used bijectiveness of mapping $\Phi$ and strong commutativity of $(\rho, \sim)$, and the rest is similar to the derivation in Section 4.2

\subsection{Proof of Theorem 8 (parallel version with strong commutativity)}

We now analyze Algorithm 2 with a deterministic choice of flaw $f$ in line 5. Equivalently, this can be viewed as running Algorithm 1 with some deterministic strategy. We will need the following result.

Lemma 23. Consider a word $W$ and a valid set of walks $\mathcal{X}$ such that $W$ is a prefix of every walk in $\mathcal{X}$. Then

$$
\sum_{\tau \in \mathcal{X}} p(\tau) \leq \gamma^{\text {init }} \cdot \lambda_{W}
$$

Proof. We use induction on $\sum_{\tau \in \mathcal{X}}(|\tau|-|W|)$. The base case $\sum_{\tau \in \mathcal{X}}(|\tau|-|W|)=0$ is straightforward: we then have $\mathcal{X} \subseteq\langle W\rangle$, and so the claim follows from Lemma 11. Consider a valid set $\mathcal{X}$ with $\sum_{\tau \in \mathcal{X}}(|\tau|-|W|) \geq 1$. Let $\hat{\tau}$ be a longest walk in $\mathcal{X}$, then $|\hat{\tau}| \geq|W|+1$. Let $\hat{\tau}^{-}$be the proper prefix of $\hat{\tau}$ of length $|\hat{\tau}|-1$. We have $\hat{\tau}^{-} \notin \mathcal{X}$ since $\mathcal{X}$ is a valid set. Define set $\mathcal{Y}$ as follows: $\mathcal{Y}=\left\{\tau \in \mathcal{X} \mid \hat{\tau}^{-}\right.$is a proper prefix of $\left.\tau\right\}$. By the choice of $\hat{\tau}$ we get $|\tau|=|\hat{\tau}|$ for all $\tau \in \mathcal{Y}$, and so we must have $\tau=\hat{\tau}^{-} \stackrel{w}{\rightarrow} \sigma$ for some $w \in F$ and $\sigma \in \Omega$. Since set $\mathcal{Y} \subseteq \mathcal{X}$ is valid, the flaw $w$ in the expression $\tau=\hat{\tau}^{-} \stackrel{w}{\rightarrow} \sigma$ must be the same for all $\tau \in \mathcal{Y}$. Thus, $\mathcal{Y}=\left\{\hat{\tau}^{-} \stackrel{w}{\rightarrow} \sigma \mid \sigma \in Y\right\}$ for some set of flaws $Y \subseteq F$. In fact, we must have $Y \subseteq A(w, \hat{\sigma})$ where $\hat{\sigma}$ is the final state of $\hat{\tau}^{-}$.

Define $\mathcal{X}^{-}=(\mathcal{X}-\mathcal{Y}) \cup\left\{\hat{\tau}^{-}\right\}$. We have

$$
\sum_{\tau \in \mathcal{X}} p(\tau)-\sum_{\tau \in \mathcal{X}^{-}} p(\tau)=\left(\sum_{\tau \in \mathcal{Y}} p(\tau)\right)-p\left(\hat{\tau}^{-}\right)=p\left(\hat{\tau}^{-}\right) \cdot\left[\left(\sum_{\sigma \in Y} \rho(\sigma \mid w, \hat{\sigma})\right)-1\right] \leq 0
$$

It is straightforward to check that set $\mathcal{X}^{-}$is valid, and $W$ is a prefix of every walk in $\mathcal{X}^{-}$. Using the induction hypothesis for $\mathcal{X}^{-}$and the inequality above gives the claim for $\mathcal{X}$.

Consider executions of Algorithm 2 consisting of at least $s$ rounds. For each such execution let $\tau$ be the walk containing flaws addressed in the first $s-1$ rounds and the first flaw addressed in round $s$. Let $\operatorname{BadPar}(s)$ be the set of such walks $\tau$.

We say that a word $U=u_{1} \ldots u_{s}$ is a chain of a walk $\tau \doteq w_{1} \ldots w_{t}$ if $U$ is a subsequence of the sequence $w_{1} \ldots w_{t}$ and $u_{i} \cong u_{i+1}$ for $i \in[s-1]$.

Proposition 24. (a) For each $\tau \in \operatorname{BadPar}(s)$ the length of a longest chain in $\tau$ equals s. (b) Set $\operatorname{BadPar}(s)$ is valid. 
Proof. Let $I_{r}$ be the set of flaws addressed in round $r$ (for $r \in[s-1]$ ), and let $I_{s}=\{f\}$ where $f$ is the first flaw addressed in round $s$. In the proof of Proposition 17 we showed that $\left(I_{1}, \ldots, I_{s}\right)$ is a stable sequence and $\tau \doteq W_{1} \ldots W_{s}$ where $W_{r}$ is a word containing the flaws in $I_{r}$ in some order (with $\left|W_{r}\right|=\left|I_{r}\right|$ ). These facts imply part (a). Let us prove (b). By construction, all walks in $\operatorname{BadPar}(s)$ follow the same deterministic strategy used in Algorithm 2. Now consider a walk $\tau^{\prime} \in \operatorname{BadPar}(s)$. By the definition of $\tau^{\prime} \in \operatorname{BadPar}(s)$, any proper prefix $\tau$ of $\tau^{\prime}$ corresponds to an execution of Algorithm 2 with at most $r-1$ rounds, and so the length of a longest chain in $\tau$ is at most $r-1$. Thus, $\tau \notin \operatorname{BadPar}(s)$.

Fix a flaw $f \in F$, and let $\operatorname{BadPar}^{f}(s)$ be the set of walks $\tau \in \operatorname{BadPar}(s)$ that contain a chain of length $s$ that ends with $f$. We now apply Theorem 21 with the set $\mathcal{X}=\operatorname{BadPar}^{f}(s)$. The set $\mathcal{X}_{\pi}$ constructed in the theorem will be denoted as $\operatorname{BadPar}_{\pi}^{f}(s)$. Since every walk $\tau \in \operatorname{BadPar}^{f}(s)$ has a chain of length $s$ that ends with $f$, so does every walk $\tau \in \operatorname{BadPar}_{\pi}^{f}(s)$ (applying a swapping mapping to $\tau$ does not affect the chain). This means that $|\operatorname{PREFIX} f(\tau)| \geq s$ for each $\tau \in \operatorname{BadPar}_{\pi}^{f}(s)$.

For a word $W \in \operatorname{Stab}_{\pi}^{\text {rev }}(\{f\}, s)$ let $\langle W \mid \neg f\rangle$ be the set of walks $\tau \in \operatorname{BadPar}_{\pi}^{f}(s)$ of the form $\tau \doteq W U$ where $U$ does not contain $f$. Note that $W$ ends with $f$, and thus $\operatorname{PREFIX}^{f}(\tau)=W$ for any $\tau \in\langle W \mid \neg f\rangle$. Theorem 21(i) gives that $\operatorname{BadPar}_{\pi}^{f}(s)=\bigcup_{W \in \operatorname{Stab}_{\pi}^{\mathrm{rev}}(\{f\}, s)}\langle W \mid \neg f\rangle$. Also, from Theorem 21(ii) we conclude that $\langle W \mid \neg f\rangle$ is a valid set for any $W \in \operatorname{Stab}_{\pi}^{\text {rev }}(\{f\}, s)$. We can thus write

$$
\begin{aligned}
& \sum_{\tau \in \operatorname{BadPar}^{f}(s)} p(\tau) \stackrel{\text { (a) }}{=} \sum_{\tau \in \operatorname{BadPar}_{\pi}^{f}(s)} p(\tau)=\sum_{W \in \operatorname{Stab}_{\pi}^{\text {rev }}(\{f\}, s)} \sum_{\tau \in\langle W \mid \neg f\rangle} p(\tau) \\
& \stackrel{\text { (b) }}{\leq} \sum_{W \in \operatorname{Stab}_{\pi}^{\text {rev }}(\{f\}, s)} \gamma^{\text {init }} \cdot \lambda_{W} \stackrel{(\text { c) }}{\leq} \gamma^{\text {init }} \cdot \mu(\{f\}) \cdot \theta^{s}
\end{aligned}
$$

where in (a) we used bijectiveness of $\Phi$ and strong commutativity of $(\rho, \sim)$, in (b) we used Lemma 23 , and in (c) we used Theorem 16. By Proposition 24, $\operatorname{BadPar}(s)=\bigcup_{f \in F} \operatorname{BadPar}^{f}(s)$, therefore

$$
\begin{aligned}
\operatorname{Pr}[\# \text { rounds } \geq s] & \leq \sum_{\tau \in \operatorname{BadPar}(s)} p(\tau) \\
& \leq \sum_{f \in F} \sum_{\tau \in \operatorname{BadPar}^{f}(s)} p(\tau) \leq \sum_{f \in F} \gamma^{\text {init }} \cdot \mu(\{f\}) \cdot \theta^{s}=\theta^{s-T}
\end{aligned}
$$

where $T$ is given by the expression in (6).

\subsection{Proof of Theorem 20(a) (swapping mapping for the atomic case)}

Recall that in the atomic case the walks can be denoted as $\tau=W[\sigma]$, since $\tau$ can be uniquely reconstructed from the word $W$ and the state $\sigma$ (see Section 5.1).

We can extend a "valid swap" operation to words in a natural way: it is a transformation of the form $\ldots f g \ldots \mapsto \ldots g f \ldots$ where $f \not g$. We will write $W \equiv W^{\prime}$ if $W^{\prime}$ can be obtained from $W$ via a sequence of valid swaps. Clearly, " $\equiv "$ is an equivalence relation. It can be seen that a walk $\tau=W[\sigma]$ can be transformed to a walk $\tau^{\prime}=W^{\prime}[\sigma]$ if and only if $W \equiv W^{\prime}$. In this case we will write $\tau^{\prime} \equiv \tau$; again, " $\equiv$ " is an equivalence relation on the set of walks.

Lemma 25. For any word $W$ there exists a sequence of valid swaps that transforms $W$ to a $\pi$-stable word. 
Proof. It suffices to show that $W$ can be transformed to a stable word via valid swaps. (Transforming a stable word $W$ to a $\pi$-stable word is straightforward: if $W=W_{1} \ldots W_{s}$ is the partition described in Definition 14 then we simply need to apply swaps inside each word $W_{r}$ to "sort" it according to $\preceq_{\pi}$; any such swap will be valid by the property of $W_{r}$ ).

Let $W=\hat{w}_{1} \ldots \hat{w}_{t}$. We will prove by induction on $i=1, \ldots, t$ that $W$ can be transformed via valid swaps to a word $W^{\prime}=w_{1} \ldots w_{i} \ldots w_{t}$ such that the prefix $w_{1} \ldots w_{i}$ is a stable word. The base case $i=1$ is trivial. Suppose the claim holds for $i-1$, and let us show it for $i \in[2, t]$.

By the induction hypothesis, $W$ can be transformed to a word $W^{\prime}=w_{1} \ldots w_{i-1} f \ldots w_{t}$ such that $w_{1} \ldots w_{i-1}$ is a stable word. Let $w_{1} \ldots w_{i-1}=W_{1} \ldots W_{s}$ be the corresponding partition described in Definition 14. Let $W_{r}$ be the rightmost word that contains a flaw $g$ with $f \cong g$. (If such word doesn't exist then we set $r=0$; thus, $r \in[0, s]$ ). If $r=s$ then we can leave the word $W^{\prime}$ as it is - it satisfies the induction hypothesis for $i$.

Suppose that $r \in[0, s-1]$. Then we repeatedly swap $f$ with the left neighbor, stopping when $f$ gets between words $W_{r}$ and $W_{r+1}$ (or at the first position, if $r=0$ ). By the definition of $r$, these are valid swaps. The new word now satisfies the induction hypothesis for $i$ (flaw $f$ will be assigned to word $\left.W_{r+1}\right)$.

The lemma means that there exists a swapping mapping $\Phi$ that sends any walk $\tau=W[\sigma]$ to a $\pi$-stable walk $\tau^{\prime}=W^{\prime}[\sigma]$. Next, we will show that any such mapping is injective on $\mathcal{X}$.

Lemma 26. Suppose that $\left\{\tau, \tau^{\prime}\right\}$ is a valid set of walks and $\tau \equiv \tau^{\prime}$. Then $\tau=\tau^{\prime}$.

Proof. We use induction on the length of $\tau$. The base case is trivial: if $|\tau|=\left|\tau^{\prime}\right|=0$ then condition $\tau \equiv \tau^{\prime}$ implies that $\tau=\tau^{\prime}=[\sigma]$ for some state $\sigma$.

Suppose that $\tau=f W[\sigma]$. Condition $\tau \equiv \tau^{\prime}$ means that $\tau^{\prime}$ and $\tau$ start at the same state. Therefore, since $\left\{\tau, \tau^{\prime}\right\}$ is a valid set, the first flaw addressed in $\tau^{\prime}$ is the same as in $\tau$. Thus, $\tau^{\prime}=f W^{\prime}[\sigma]$. The set $\left\{W[\sigma], W^{\prime}[\sigma]\right\}$ must be valid (since the set $\left\{f W[\sigma], f W^{\prime}[\sigma]\right\}$ is valid). We will show below that $W[\sigma] \equiv W^{\prime}[\sigma]$; this will mean that $W[\sigma]=W^{\prime}[\sigma]$ by the induction hypothesis, thus giving the desired result.

We need to show that $W \equiv W^{\prime}$. We know that $f W \equiv f W^{\prime}$, therefore there exists a sequence of words $U^{(1)}, \ldots, U^{(k)}$ with $U^{(1)}=f W, U^{(k)}=f W^{\prime}$ such that $U^{(i+1)}$ is obtained from $U^{(i)}$ via a single valid swap operation. Let $V^{(i)}$ be the word obtained from $U^{(i)}$ by moving the first occurence of flaw $f$ in $U^{(i)}$ to the first position. Thus, for each $i \in[k]$ we have $V^{(i)}=f W^{(k)}$ for some word $W^{(k)}$. It can be seen that either $V^{(i+1)}=V^{(i)}$ (if the valid swap operation applied to $U^{(i)}$ involved the first occurence of $f$ ) or $V^{(i+1)}$ is obtained from $V^{(i)}$ via a valid swap (otherwise). Thus, either $W^{(i+1)}=W^{(i)}$ or $W^{(i+1)}$ is obtained from $W^{(i)}$ via a valid swap. It remains to notice that $W^{(1)}=W$ and $W^{(k)}=W^{\prime}$.

We can now prove that $\Phi$ is injective on $\mathcal{X}$. Suppose that $\Phi(\tau)=\Phi\left(\tau^{\prime}\right)$ for walks $\tau, \tau^{\prime} \in \mathcal{X}$. We have $\tau \equiv \Phi(\tau)=\Phi\left(\tau^{\prime}\right) \equiv \tau^{\prime}$, and so $\tau \equiv \tau^{\prime}$. From the previous lemma we obtain that $\tau=\tau^{\prime}$, which concludes the proof of Theorem 20(a).

\subsection{Proof of Theorems 20(b) and 21 (swapping mappings for the non-atomic case)}

Consider a word $W=w_{1} \ldots w_{t}$. It will be convenient to alternatively write it as $W=\mathbf{w}_{1} \ldots \mathbf{w}_{t}$ where $\mathbf{w}_{i}=\left(w_{i}, n_{i}\right)$ and $n_{i}$ counts from the left which occurrence of the flaw $w_{i}$ it is: $n_{i}=\mid\{j \in$ $\left.[i] \mid w_{j}=w_{i}\right\} \mid \geq 1$. Note that all elements $\mathbf{w}_{1}, \ldots, \mathbf{w}_{t}$ are distinct. Tuple $\mathbf{w}_{i}$ will be called a named flaw. The flaw associated with the named flaw $\mathbf{f}$ will be denoted without the bold font as $f$, i.e. 
$\mathbf{f}=\left(f, n_{f}\right)$. We say that a word $W=\mathbf{w}_{1} \ldots \mathbf{w}_{t}$ over named flaws has consistent counts if it is constructed from the unnamed version $w_{1} \ldots w_{t}$ as described above.

We will denote the element of $F$ fixed in Theorem 21 as $\hat{f}$ (rather than $f$ ). In the case of Theorem 20(b) element $\hat{f}$ is not defined; to indicate this fact, we will write $\hat{f}=\perp$.

For a walk $\tau \doteq \mathbf{w}_{1} \ldots \mathbf{w}_{t}$ let us define a directed acyclic graph $\mathbf{G}_{\tau}=\left(\mathbf{V}_{\tau}, \mathbf{E}_{\tau}\right)$ as follows: its nodes are $V_{\tau}=\left\{\mathbf{w}_{1}, \ldots, \mathbf{w}_{t}\right\}$, and the set $\mathbf{E}_{\tau}$ contains all edges of the form $(\mathbf{f}, \mathbf{g})$ where $f \cong g$ and f occurs in $\tau$ before g, i.e. $\tau \stackrel{\bullet}{=}$. f ...g . ...

We say that "walk $\tau$ contains $\hat{f}$ " if either (i) $\hat{f}=\perp$, or (ii) $\hat{f} \neq \perp$ and $\tau \doteq \ldots \hat{f} \ldots$. For a walk $\tau$ containing $\hat{f}$ we make the following definitions:

- If $\hat{f}=\perp$ (i.e. in the case of Theorem $20(\mathrm{~b})$ ), let $\hat{\mathbf{V}}_{\tau}=\mathbf{V}_{\tau}$. For $\mathbf{f} \in \hat{\mathbf{V}}_{\tau}$ let $d_{\tau}(\mathbf{f})$ be the length of the longest path from $\mathbf{f}$ to a sink of $\mathbf{G}_{\tau}$ plus one (so that for any sink node $\mathbf{f} \in \mathbf{V}_{\tau}$ we have $\left.d_{\tau}(\mathbf{f})=1\right)$.

- If $\hat{f} \neq \perp$ (i.e. in the case of Theorem 21), let $\hat{\mathbf{V}}_{\tau}$ be the set of flaws $\mathbf{g} \in \mathbf{V}_{\tau}$ from which node $\hat{\mathbf{f}}$ can be reached in $\mathbf{G}_{\tau}$, where $\hat{\mathbf{f}}$ is the named flaw corresponding to the rightmost occurence of $\hat{f}$ in $\tau$. For $\mathbf{f} \in \hat{\mathbf{V}}_{\tau}$ let $d_{\tau}(\mathbf{f})$ be the length of the longest path from $\mathbf{f}$ to $\hat{\mathbf{f}}$ in $\mathbf{G}_{\tau}$ plus one (so that $\left.d_{\tau}(\hat{\mathbf{f}})=1\right)$.

Furthermore, we define word $\Psi(\tau)$ as follows:

- For an integer $r \geq 1$ let $\mathbf{I}_{r}=\left\{\mathbf{f} \in \hat{\mathbf{V}}_{\tau} \mid d_{\tau}(\mathbf{f})=r\right\}$, and let $W_{r}$ be the word consisting of the named flaws in $\mathbf{I}_{r}$ sorted in the decreasing order (with respect to $\preceq_{\pi}$ ). 4 Then $\Psi(\tau)=W_{s} \ldots W_{1}$ where $s=\max \left\{d_{\tau}(\mathbf{f}) \mid \mathbf{f} \in \hat{\mathbf{V}}_{\tau}\right\}$.

Lemma 27. The word $W=\Psi(\tau)$ is reversely $\pi$-stable. Also, $R_{W}^{\mathrm{rev}}=\{\hat{f}\}$ if $\hat{f} \neq \perp$.

Proof. Let $I_{r}=\left\{f \mid \mathbf{f} \in \mathbf{I}_{r}\right\}$ be the "unnamed" version of the set $\mathbf{I}_{r}$. Consider $r \in[s-1]$. From the the definition of $\mathbf{I}_{r}$ and $\mathbf{I}_{r+1}$ we obtain that for every $\mathbf{f} \in \mathbf{I}_{r+1}$ there exists $\mathbf{g} \in \mathbf{I}_{r}$ with $(\mathbf{f}, \mathbf{g}) \in E_{\tau}$ (implying that $f \in \Gamma^{+}(g)$ ). This gives that $I_{r+1} \subseteq \Gamma^{+}\left(I_{r}\right)$. Also, if $\hat{f} \in F$ then we have $\mathbf{I}_{1}=\{\hat{\mathbf{f}}\}$ and so $I_{1}=\{\hat{f}\}$. Observing that $W=W_{s} \ldots W_{1}$ we obtain the desired claims.

It is straighforward to check that applying valid swaps to $\tau$ does not affect graph $\mathbf{G}_{\tau}$, set $\hat{\mathbf{V}}_{\tau}$ and word $\Psi(\tau)$. Our goal will be to apply swaps to $\tau$ so that word $\Psi(\tau)$ becomes a prefix of $\tau$. We will do this by applying swaps to swappable pairs in $\tau$.

Definition 28. Consider a walk $\tau$ containing $\hat{f}$. A pair $(\mathbf{f}, \mathbf{g})$ of named flaws is called a swappable pair in $\tau$ if it can be swapped in $\tau$ (i.e. $\tau \doteq \ldots$ fg ... and $f \neq g$ ) and either

(i) $(\mathbf{f}, \mathbf{g}) \in\left(\mathbf{V}_{\tau}-\hat{\mathbf{V}}_{\tau}\right) \times \hat{\mathbf{V}}_{\tau}$, or

(ii) $(\mathbf{f}, \mathbf{g}) \in \hat{\mathbf{V}}_{\tau} \times \hat{\mathbf{V}}_{\tau}$ and their order in $\Psi(\tau)$ is different: $\Psi(\tau)=\ldots \mathbf{g} \ldots \mathbf{f} \ldots$.

The position of the rightmost swappable pair in $\tau$ will be denoted as $k(\tau)$, where the position of $(\mathbf{f}, \mathbf{g})$ in $\tau$ is the number of named flaws that precede $\mathbf{g}$ in $\tau$. If $\tau$ does not contain a swappable pair then $k(\tau)=0$. Thus, $k(\tau) \in[0,|\tau|-1]$.

It can be seen that the procedure of repeatedly applying swaps to swappable pairs in $\tau$ must terminate. Indeed, swapping a pair of the form (i) moves a named flaw in $\hat{\mathbf{V}}_{\tau}$ to the left, which can happen only a finite number of times. Similarly, swapping a pair of the form (ii) decreases the number of pairs $\{\mathbf{f}, \mathbf{g}\} \in\left(\begin{array}{c}\hat{\mathbf{V}}_{\tau} \\ 2\end{array}\right)$ whose relative order in $\tau$ is not consistent with the relative order in $\Psi(\tau)$, which again can happen only a finite number of times. Note, swaps of the other type do not affect these arguments.

\footnotetext{
${ }^{4}$ It can be seen that we have $f \neq g$ for any distinct $\mathbf{f}, \mathbf{g} \in \mathbf{I}_{r}$. Therefore, "sorting $\mathbf{I}_{r}$ in the decreasing order" in the definition is a valid operation (where we assume that $\mathbf{f} \prec_{\pi} \mathbf{g}$ if $f \prec_{\pi} g$ ).
} 
Lemma 29. Consider a walk $\tau \doteq A U$ containing $\hat{f}$ where $A, U$ are some words, and there are no swappable pairs inside $U$. Then $U=B C$ where sequence $B$ is a subsequence of $\Psi(\tau)$, and $C$ does not contain named flaws from $\hat{\mathbf{V}}_{\tau}$.

In particular, if $|A|=0$ and $\tau \doteq U$ does not contain a swappable pair then $\tau \doteq \Psi(\tau) C$.

Proof. Let $\mathbf{u}_{1}, \ldots, \mathbf{u}_{m}$ be the named flaws from $\hat{\mathbf{V}}_{\tau}$ that occur in $U$ (listed in the order of their appearance in $U$ ). We claim that $\mathbf{u}_{1} \ldots \mathbf{u}_{m}$ is a prefix of $U$. Indeed, suppose not, then $U=\ldots \mathbf{f} \mathbf{u}_{i} \ldots$ where $\mathbf{f} \notin \hat{\mathbf{V}}_{\tau}$ and $\mathbf{u}_{i} \in \hat{\mathbf{V}}_{\tau}$. This means that $\left(\mathbf{f}, \mathbf{u}_{i}\right) \notin E_{\tau}$, and so $f \neq u_{i}$. But then (f, $\left.\mathbf{u}_{i}\right)$ is a swappable pair in $U$ - a contradiction.

We obtained a decomposition $\tau \doteq A B C$ where $B=\mathbf{u}_{1} \ldots \mathbf{u}_{m}$. It remains to show that $B$ is a subsequence of $\Psi(\tau)$. For that it suffices to prove that for any $i \in[m-1]$ the relative order of $\mathbf{u}_{i}$ and $\mathbf{u}_{i+1}$ in $B$ is the same as in $\Psi(\tau)$, i.e. $\Psi(\tau)=\ldots \mathbf{u}_{i} \ldots \mathbf{u}_{i+1} \ldots$ We must have $u_{i} \cong u_{i+1}$ (otherwise $\left(\mathbf{u}_{i}, \mathbf{u}_{i+1}\right)$ would be a swappable pair in $\tau$, contradicting the assumption). Therefore, $\left(\mathbf{u}_{i}, \mathbf{u}_{i+1}\right) \in \mathbf{E}_{\tau}$, implying that $d_{\tau}\left(\mathbf{u}_{i}\right)>d_{\tau}\left(\mathbf{u}_{i+1}\right)$. Inspecting the definition of $\Psi(\tau)$, we conclude that $\mathbf{u}_{i}$ should be to the left of $\mathbf{u}_{i+1}$ in $\Psi(\tau)$, which is what we needed to show.

To summarize, we showed that taking a walk $\tau \in \mathcal{X}$ and repeatedly applying swaps to swappable pairs terminates and produces a walk $\tau^{\prime}$ such that the word $\Psi\left(\tau^{\prime}\right)=\Psi(\tau)$ is a prefix of $\tau^{\prime}$. One consequence is that $\Psi(\tau)$ must have consistent counts (since applying valid swaps preserves consistency of counts). We can also conclude that there exists a swapping mapping $\Phi$ and set $\mathcal{X}_{\pi}=\Phi(\mathcal{X})$ with the following properties: in the case of Theorem 20(b) all walks $\tau \in \mathcal{X}_{\pi}$ are reversely $\pi$-stable, and in the case of Theorem 21 set $\mathcal{X}_{\pi}$ satisfies condition (i). It remains to show that $\Phi$ can be chosen so that it is injective on $\mathcal{X}$, and in the case of Theorem 21 set $\mathcal{X}_{\pi}$ satisfies condition (ii). For that we need to be careful with the order in which we apply swaps to swappable pairs. We will use the following algorithm. First, let $\mathcal{X}_{0}=\mathcal{X}$, and then for $p=0,1,2, \ldots$ do the following:

- Let $k=\max _{\tau \in \mathcal{X}_{p}} k(\tau)$. If $k=0$ then terminate.

- For each $\tau \in \mathcal{X}_{p}$ do the following: if $k(\tau)=k$ then swap the pair (f, g) at position $k$ in $\tau$, otherwise leave $\tau$ unchanged. Let $\mathcal{X}_{p+1}$ be the new set of walks.

Note that this algorithm defines a mapping from $\mathcal{X}_{p}$ to $\mathcal{X}_{p+1}$ in a natural way. Let us fix a word $W=\mathbf{w}_{1} \ldots \mathbf{w}_{t}$ over named flaws with consistent counts, and define $\mathcal{X}_{p}[W]=\left\{\tau \in \mathcal{X}_{p} \mid \Psi(\tau)=W\right\}$ for an index $p \geq 0$. Since applying valid swaps to $\tau$ does not affect $\Psi(\tau)$, the mapping defined by the algorithm above sends walks in $\mathcal{X}_{p}[W]$ to walks in $\mathcal{X}_{p+1}[W]$. The remaining claims will follow from the following result (note that the set $\mathcal{X}_{0}[W] \subseteq \mathcal{X}$ is valid by the assumption of the theorems).

Lemma 30. If set $\mathcal{X}_{p}[W]$ is valid then so is $\mathcal{X}_{p+1}[W]$, and the mapping from $\mathcal{X}_{p}[W]$ to $\mathcal{X}_{p+1}[W]$ defined by the algorithm above is injective.

Proof. Suppose that the lemma is false. This means that there exist distinct walks $\tau, \bar{\tau} \in \mathcal{X}_{p}[W]$ that were transformed to walks $\eta, \bar{\eta} \in \mathcal{X}_{p+1}[W]$, respectively, such that $\eta \wedge \bar{\eta}=\ldots[\Omega]$ (violating either injectiveness if $\eta=\bar{\eta}$, or validity if $\eta \neq \bar{\eta}$ ). At least one of the walks $\tau, \bar{\tau}$ must have changed; assume w.l.o.g. that the walk $\tau$ has changed, i.e. $\eta \neq \tau$. We thus have

$$
\tau \stackrel{\bullet}{ } \xi \mathbf{f} \mathbf{g} X, \quad \eta \stackrel{\bullet}{=} \mathbf{g} \mathbf{f} X
$$

where $\xi$ is some walk (of length $k-1=k(\tau)-1$ ), (f, $\mathbf{g})$ is a swappable pair in $\tau$, and $X$ is some word over named flaws. Note that $\bar{\eta}$ either equals $\bar{\tau}$ or is obtained from $\bar{\tau}$ by swapping a pair at position $k$ in $\bar{\tau}$. 
We have $\tau \wedge \bar{\tau} \neq \ldots[\Omega]$ since $\mathcal{X}_{p}[W]$ is valid. If $\tau \wedge \bar{\tau}$ is a proper prefix of $\xi$ then $\eta \wedge \bar{\eta}=\tau \wedge \bar{\tau}$, contradicting the assumption that $\eta \wedge \bar{\eta}=\ldots[\Omega]$. Thus, $\xi$ is a prefix of $\tau \wedge \bar{\tau}$. Condition $\tau \wedge \bar{\tau} \neq \ldots[\Omega]$ means that

$$
\bar{\tau} \doteq \xi \mathbf{f} Y
$$

for some word $Y$ over named flaws. By construction, walk $\tau$ does not have swappable pairs inside $\mathrm{g} X$, and walk $\bar{\tau}$ does not have swappable pairs inside $Y$. Lemma 29 gives that $\mathrm{g} X=B C$ and $Y=\bar{B} \bar{C}$ where

(i) $B, \bar{B}$ are subsequences of $\Psi(\tau)=\Psi(\bar{\tau})=W$ (with $B=\mathbf{g} \ldots$ ), and

(ii) $C, \bar{C}$ do not contain named flaws from $\hat{\mathbf{V}}$ where we denoted $\hat{\mathbf{V}}=\hat{\mathbf{V}}_{\tau}=\hat{\mathbf{V}}_{\bar{\tau}}$ (it is the set of named flaws present in $W$ ).

We obtained that $\tau \doteq \xi \mathbf{f} B C$ and $\bar{\tau} \doteq \xi \mathbf{f} \bar{B} \bar{C}$. The set of named flaws present in $B$ must be the same as the set of named flaws present in $\bar{B}$ (namely, it is $\hat{\mathbf{V}}$ minus the set of named flaws present in the sequence $\xi \mathbf{f}$ ). Furthermore, $B$ and $\bar{B}$ are subsequences of $W$, so we must have $B=\bar{B}$ (recall that all named flaws in $W$ are unique). Therefore, $\bar{\tau} \doteq \xi \mathbf{f} \mathbf{g} \ldots$ This means that (f, $\mathbf{g})$ is a swappable pair in $\bar{\tau}$, and $\bar{\eta}$ is obtained from $\bar{\tau}$ by swapping $\mathbf{f}$ and $\mathbf{g}$.

To summarize, we showed that walks $\tau, \bar{\tau}, \eta, \bar{\eta}$ have the following forms:

$$
\begin{aligned}
\tau & =\xi\left[\sigma_{1}\right] f\left[\sigma_{2}\right] g\left[\sigma_{3}\right] \zeta & & =\xi\left[\sigma_{1}\right] g\left[\sigma_{2}^{\prime}\right] f\left[\sigma_{3}\right] \zeta \\
\bar{\tau} & =\xi\left[\sigma_{1}\right] f\left[\bar{\sigma}_{2}\right] g\left[\bar{\sigma}_{3}\right] \bar{\zeta} & & \bar{\eta}=\xi\left[\sigma_{1}\right] g\left[\bar{\sigma}_{2}^{\prime}\right] f\left[\bar{\sigma}_{3}\right] \bar{\zeta}
\end{aligned}
$$

where $\sigma_{1}$ is the last state of walk $\xi, \sigma_{3}$ is the first state of walk $\zeta$, and $\bar{\sigma}_{3}$ is the first state of walk $\bar{\zeta}$. Condition $\eta \wedge \bar{\eta}=\ldots[\Omega]$ implies that $\bar{\sigma}_{2}^{\prime}=\sigma_{2}^{\prime}$ and $\bar{\sigma}_{3}=\sigma_{3}$. Since the SWAP operation from Definition 3 is injective, we obtain that $\bar{\sigma}_{2}=\sigma_{2}$. It can now be seen that we cannot have simultaneously $\tau \wedge \bar{\tau} \neq \ldots[\Omega]$ and $\eta \wedge \bar{\eta}=\ldots[\Omega]$. We have obtained a contradiction.

\section{Examples of commutative resampling oracles for non-Cartesian spaces}

In this section we show that resampling oracles for permutations used in [9, 10] and for perfect matchings in complete graphs used in [10] are strongly commutative.

These oracles have two important properties: (i) they are atomic; (ii) $\rho\left(\sigma^{\prime} \mid f, \sigma\right)$ are uniform distributions over $A(f, \sigma)$ for any $f \in F$ and $\sigma \in f$. Distributions $\rho$ satisfying these two properties were studied by Achlioptas and Iliopoulos [1], albeit with a different terminology: they used a directed multigraph $D$ instead of $\rho$. This multigraph is defined as follows: its set of nodes is $\Omega$, and its set of edges is the set of all valid walks $\sigma \stackrel{f}{\rightarrow} \sigma^{\prime}$. (Each edge of $D$ is labeled by a flaw in $F$ ). It is atomic if any state $\sigma^{\prime} \in \Omega$ has at most one incoming edge in $D$ labeled by a given flaw $f \in F$. Note that one can recover sets $A(f, \sigma)$ from $D$, since $A(f, \sigma)=\left\{\sigma^{\prime} \mid\right.$ edge $\sigma \stackrel{f}{\rightarrow} \sigma^{\prime}$ belongs to $\left.D\right\}$. Therefore, distributions $\rho$ are uniquely defined by $D$, assuming that property (ii) holds. It can be seen that there is a one-to-one correspondence between atomic multigraphs and distributions $\rho$ satisfying (i) and (ii).

To prove commutativity of the oracles mentioned earlier, we find it easier to take an indirect approach: first, we will describe a generic route for constructing atomic multigraphs, then apply it to permutations and matchings and prove weak commutativity. We will then see that the resulting resampling procedure coincides with that in [9, 10].

The constructed multigraph will satisfy the following property: for each flaw $f$ there exists constant $A_{f}$ such that $|A(f, \sigma)|=A_{f}$ all $\sigma \in f$. Thus, $\rho\left(\sigma^{\prime} \mid f, \sigma\right)=\frac{1}{A_{f}}$ for $\sigma^{\prime} \in A(f, \sigma)$, and so weak commutativity will imply strong commutativity. 
We start with some general observations. For an atomic multigraph $D$ let us define a mapping $\psi: F \times \Omega \rightarrow \Omega \cup\{\perp\}$ that specifies a "backward step" for $f \in F$ and $\sigma^{\prime} \in \Omega$ as follows: if there

exists a (unique) state $\sigma \in \Omega$ such that $\sigma \stackrel{f}{\rightarrow} \sigma^{\prime}$ then $\psi\left(f, \sigma^{\prime}\right)=\sigma$, otherwise $\psi\left(f, \sigma^{\prime}\right)=\perp$. Note that $\psi$ satisfies the following properties:

(I) If $\psi\left(f, \sigma^{\prime}\right) \neq \perp$ then $\psi\left(f, \sigma^{\prime}\right) \in f$.

(II) For any $f \in F$ and $\sigma \in f$ there exists at least one $\sigma^{\prime} \in \Omega$ with $\psi\left(f, \sigma^{\prime}\right)=\sigma$.

It is not difficult to see that $D$ can be uniquely reconstructed from $\Omega, F$ and $\psi$, since for each $f \in F$ and $\sigma \in f$ we have $A(f, \sigma)=\left\{\sigma^{\prime} \in \Omega \mid \psi\left(f, \sigma^{\prime}\right)=\sigma\right\}$. Furthermore, any triplet $(\Omega, F, \psi)$ specifies a valid atomic multigraph $D$, as long as $\psi$ satisfies properties (I) and (II) (and $F$ is some set of non-empty subsets of $\Omega$ ). Property (II), in particular, is equivalent to the condition that $A(f, \sigma)$ is non-empty for each $f \in F$ and $\sigma \in f$.

Thus, the problem of constructing the set of actions $A(f, \sigma)$ for a given $f, \sigma$ can be shifted to the problem of constructing a mapping $\psi$. Of course, after constructing $\psi$ one still needs to show that sampling from $A(f, \sigma)$ can be done efficiently.

We remark that Definition 2 of the potential causality graph can also be reformulated in terms of the mapping $\psi$, as stated below (this claim follows directly from definitions).

Proposition 31. Undirected graph $(F, \sim)$ is a potential causality graph for $D$ if for any $f, g \in F$ with $f \nsim g$ and any $\sigma^{\prime} \in g$ with $\psi\left(f, \sigma^{\prime}\right) \neq \perp$ we have $\psi\left(f, \sigma^{\prime}\right) \in g$ and $f \neq g$.

\subsection{Matchings}

We now apply the route outlined above to some matching problems. Let $G=(V, E)$ be an undirected graph with $|V|=2 n$ nodes that satisfies the following condition:

(*) If $\left(u^{\prime}, u, v, v^{\prime}\right)$ is a path in $G$ with distinct nodes then $\left\{u^{\prime}, v^{\prime}\right\} \in E$.

We will consider the case when $\Omega$ is the set of perfect matchings in $G$ (so that each object $\sigma \in \Omega$ is a subset of $E$ ). We allow any flaw of the form $f_{M}=\{\sigma \in \Omega \mid M \subseteq \sigma\}$ where $M$ is a fixed subset of $E$. It can be assumed w.l.o.g. that $M$ is a matching (otherwise $f_{M}$ would be empty). Thus, $F$ can be any subset of $\left\{f_{M} \mid M \in \mathcal{M}\right\}$ where $\mathcal{M}$ denotes the set of matchings in $G$, with $\Omega \subset \mathcal{M}$. Two special cases of this framework have been considered [9, 1, 10]:

[P1] $G$ is the complete graph on $2 n$ vertices, so that $\Omega$ is the set of all perfect matchings of $V$.

[P2] Set $V$ can be partitioned into disjoint subsets $A_{1}, B_{1}, \ldots, A_{r}, B_{r}$ such that $\left|A_{i}\right|=\left|B_{i}\right|$ for $i \in[r]$ and $E=\left\{\{u, v\} \mid u \in A_{i}, v \in B_{i}, i \in[r]\right\}$. Thus, $G$ is a union of $r$ complete bipartite graphs, and set $\Omega$ corresponds to $r$ permutations.

In fact, these are essentially the only possibilities allowed by condition $(*)$ : it can be shown that if $G$ contains at least one perfect matching then each component of $G$ is either a complete graph $K_{m}$ or a complete bipartite graph $K_{m, m}$. We will not need this claim, and so we leave it without a proof. Instead, we just assume that one of the two cases [P1,P2] holds (but unlike previous work, we will treat them in a unified way, relying mostly on condition $(*))$.

We use the following potential causality graph: $f_{M} \sim f_{M^{\prime}}$ for $f_{M}, f_{M^{\prime}} \in F$ if $M \cup M^{\prime}$ is not a matching or $M=M^{\prime}$. This graph is the same or slightly smaller than graphs used previously for cases [P1] and [P2]. (For [P2] the works [9, 10] used a larger relation $\sim^{\prime}$ instead where $f_{M} \sim^{\prime} f_{M^{\prime}}$ if $M \cup M^{\prime}$ is not a matching or $\left.M \cap M^{\prime} \neq \varnothing\right)$.

We will construct a mapping $\hat{\psi}: \mathcal{M} \times \Omega \rightarrow \Omega$ that satisfies $M \subseteq \hat{\psi}(M, \sigma)$ for any $M \in \mathcal{M}$ and $\sigma \in \Omega$. It will correspond to the mapping $\psi$ in a natural way, i.e. $\psi\left(f_{M}, \sigma\right)=\hat{\psi}(M, \sigma)$ for $f_{M} \in F$. Clearly, such $\psi$ will be defined everywhere on $F \times \Omega$, and will satisfy property (I). 


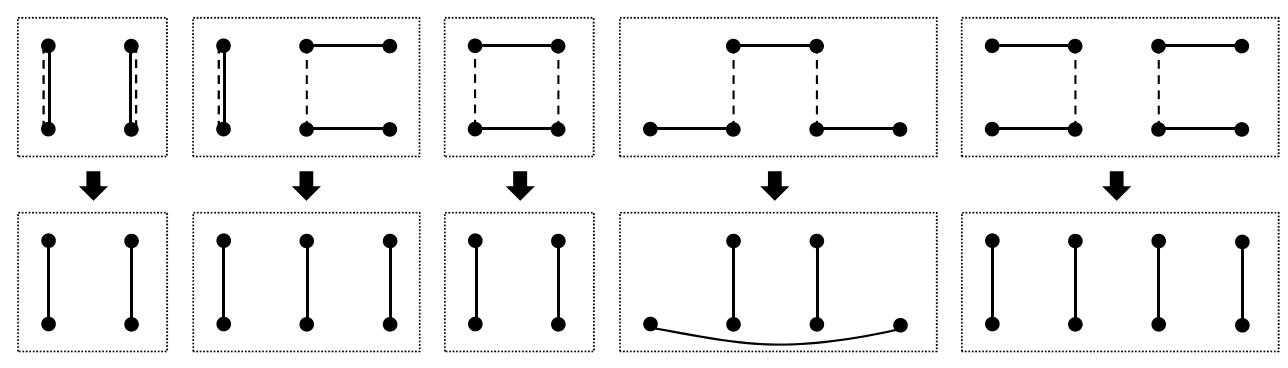

Figure 1: Possible cases for $M=\left\{e_{1}, e_{2}\right\} \in \mathcal{M}$ and $\sigma \in \Omega$. Top row: solid lines indicate edges in $\sigma$, dashed lines indicate edges in $M$. Bottom row: result of $\psi\left(\left\{e_{1}\right\}, \psi\left(\left\{e_{2}\right\}, \sigma\right)\right)=\psi\left(\left\{e_{2}\right\}, \psi\left(\left\{e_{1}\right\}, \sigma\right)\right)$.

Defining $\hat{\psi}$ Consider $M \in \mathcal{M}$ and $\sigma \in \Omega$. If $M$ contains a single edge $e=\{u, v\}$, then we find unique $u^{\prime}, v^{\prime}$ with $\left\{u, u^{\prime}\right\},\left\{v, v^{\prime}\right\} \in \sigma$, and set

$$
\hat{\psi}(\{e\}, \sigma)=\left(\sigma-\left\{\left\{u, u^{\prime}\right\},\left\{v, v^{\prime}\right\}\right\}\right) \cup\left\{\{u, v\},\left\{u^{\prime}, v^{\prime}\right\}\right\}
$$

Note that if $\{u, v\} \in \sigma^{\prime}$ then $\hat{\psi}(M, \sigma)=\sigma$. Otherwise nodes $u, v, u^{\prime}, v^{\prime}$ are distinct, and we have $\hat{\psi}(M, \sigma) \in \Omega$ by the assumption $(*)$ on the graph $G$.

Now suppose that $M=\left\{e_{1}, \ldots, e_{k}\right\}$ contains more than one edge. Then we define

$$
\sigma_{0}=\sigma \quad \sigma_{1}=\hat{\psi}\left(\left\{e_{1}\right\}, \sigma_{0}\right) \quad \sigma_{2}=\hat{\psi}\left(\left\{e_{2}\right\}, \sigma_{1}\right) \quad \ldots \quad \sigma_{k}=\hat{\psi}\left(\left\{e_{k}\right\}, \sigma_{k-1}\right)
$$

and set $\hat{\psi}(M, \sigma)=\sigma_{k}$. To show that this is well-defined, we need to prove that the result does not depend on the chosen ordering of $M$. It suffices to prove this claim for $|M|=2$, then we can use an induction argument (since any ordering of $M$ can be transformed to any other ordering via a sequence of operations that swap adjacent elements). Proving it for $|M|=2$ can be done by inspecting all possible cases, which are visualized in Fig. 1; verification of the claim in each case is left to the reader.

Proposition 32. (a) In (14) we have $\left\{e_{1}, \ldots, e_{i}\right\} \subseteq \sigma_{i}$ for any $i \in[k]$. Consequently, $M \subseteq \hat{\psi}(M, \sigma)$ for any $M \in \mathcal{M}$ and $\sigma \in \Omega$ (and so property (I) holds).

(b) For any $\sigma \in \Omega$ and $M, M^{\prime} \in \mathcal{M}$ with $M \cup M^{\prime} \in \mathcal{M}$ we have $\hat{\psi}\left(M, \hat{\psi}\left(M^{\prime}, \sigma\right)\right)=\hat{\psi}\left(M^{\prime}, \hat{\psi}(M, \sigma)\right)$. Consequently, the multigraph $D$ defined by $(\Omega, F, \psi)$ is weakly commutative with respect to $\sim$.

(c) Relation $\sim$ is a potential causality graph for $D$.

Proof. (a) We can use induction on $i$. The base case $i=0$ is vacuous. The induction step for $i$ follows from the definition of the mapping $\hat{\psi}\left(\left\{e_{i}\right\}, \sigma_{i-1}\right)$ and the fact that $\sigma_{i-1}$ cannot contain edges that connect an endpoint of $e_{i}$ to an endpoint of $e_{j}$ for $j \in[i-1]$ (since $\left\{e_{1}, \ldots, e_{i-1}\right\} \subseteq \sigma_{i-1}$ by the induction hypothesis, and $\left\{e_{1}, \ldots, e_{i}\right\}$ is a matching).

(b) We claim $\hat{\psi}\left(M^{\prime}, \hat{\psi}(M, \sigma)\right)=\hat{\psi}\left(M \cup M^{\prime}, \sigma\right)$. Indeed, let $M=\left\{e_{1}, \ldots, e_{\ell}\right\}$ and $M^{\prime}=$ $\left\{e_{\ell+1}, \ldots, e_{k}\right\}$. Define $\sigma_{0}, \sigma_{1}, \ldots, \sigma_{k}$ as in (14), then $\hat{\psi}(M, \sigma)=\sigma_{\ell}$ and $\hat{\psi}\left(M^{\prime}, \sigma_{\ell}\right)=\sigma_{k}$. Thus, it remains to show that $\hat{\psi}\left(M \cup M^{\prime}, \sigma\right)=\sigma_{k}$. For that we need to observe that if some edge $e_{i}$ appears in the sequence $\left(e_{1}, \ldots, e_{k}\right)$ the second time then we have $e_{i} \in \sigma_{i-1}$ and consequently $\sigma_{i}=\hat{\psi}\left(\left\{e_{i}\right\}, \sigma_{i-1}\right)=\sigma_{i-1}$. Thus, such $e_{i}$ can be removed from the sequence without affecting the result. After removing duplicates we conclude that $\hat{\psi}\left(M \cup M^{\prime}, \sigma\right)=\sigma_{k}$ by the definition of $\hat{\psi}$.

In a similar way we can show that $\hat{\psi}\left(M, \hat{\psi}\left(M^{\prime}, \sigma\right)\right)=\hat{\psi}\left(M \cup M^{\prime}, \sigma\right)$. This proves the claim. (c) Let us show that conditions of Proposition 31 hold. Consider flaws $f=f_{M}, g=f_{M^{\prime}}$ in $F$ with $f_{M} \nsim f_{M^{\prime}}$ and object $\sigma \in f_{M^{\prime}}$. Condition $f_{M} \neq f_{M^{\prime}}$ holds since $f_{M} \nsim f_{M^{\prime}}$, so we need to show that $\psi\left(f_{M}, \sigma\right) \in f_{M^{\prime}}$, or equivalently $M^{\prime} \subseteq \hat{\psi}(M, \sigma)$. 
Assume that $M=\left\{e_{1}, \ldots, e_{k}\right\}$, and define sequence $\sigma_{0}, \sigma_{1}, \ldots, \sigma_{k-1}, \sigma_{k}=\hat{\psi}(M, \sigma)$ as in (14). Indeed, for the base case the claim $M^{\prime} \subseteq \sigma$ holds since $\sigma \in f_{M^{\prime}}$, and for the induction step we need to use the definition of $\hat{\psi}$ and the fact that $M \cup M^{\prime} \in \mathcal{M}$ (which holds since $f_{M} \nsim f_{M^{\prime}}$ ). We leave verification of the induction step to the reader.

Sampling from $A\left(f_{M}, \sigma_{k}\right)$ The general idea is to "reverse" the process in eq. (14): given flaw $f_{M}$ with $M=\left\{e_{1}, \ldots, e_{k}\right\} \in \mathcal{M}$ and object $\sigma_{k} \in f_{M}$, we first generate possible values for $\sigma_{k-1}$, then for $\sigma_{k-2}$, and so on.

For a subset $S \subseteq E$ let $\vec{S}=\{(u, v),(v, u) \mid\{u, v\} \in A\}$ be a "directed copy" of $S$. For an object $\sigma \in \Omega$ and edges $(u, v),\left(u^{\prime}, v^{\prime}\right) \in \vec{\sigma}$ define

$$
\operatorname{Swap}_{\sigma}\left((u, v),\left(u^{\prime}, v^{\prime}\right)\right)=\left(\sigma-\left\{\{u, v\},\left\{u^{\prime}, v^{\prime}\right\}\right\}\right) \cup\left\{\left\{u, u^{\prime}\right\},\left\{v, v^{\prime}\right\}\right\}
$$

Finally, for an object $\sigma \in \Omega$ and an edge $(u, v) \in \vec{\sigma}$ let us define

$$
\mathcal{N}_{\sigma}(u, v)=\left\{\left(u^{\prime}, v^{\prime}\right) \in \vec{\sigma} \mid \operatorname{Swap}_{\sigma}\left((u, v),\left(u^{\prime}, v^{\prime}\right)\right) \in \Omega\right\}
$$

It can be checked that $(v, u) \in \mathcal{N}_{\sigma}(u, v)$ and $(u, v) \notin \mathcal{N}_{\sigma}(u, v)$. Furthermore, in the special cases above we have the following:

$[\mathrm{P} 1] \mathcal{N}_{\sigma}(u, v)=\vec{\sigma}-\{(u, v)\}$.

[P2] If $(u, v) \in A_{i} \times B_{i}$ then $\mathcal{N}_{\sigma}(u, v)=\left(B_{i} \times A_{i}\right) \cap \vec{\sigma}$.

We can now formulate the sampling algorithm (see Algorithm 3).

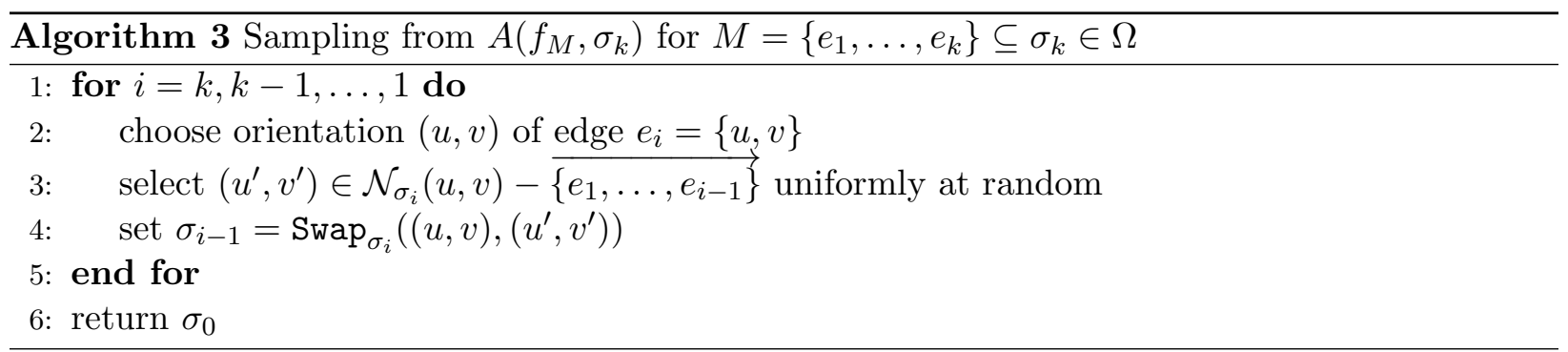

Let us verify the correctness of this algorithm. Using the definitions of $\hat{\psi}$ and $\operatorname{Swap}_{\sigma}$, the following fact can be easily checked.

Lemma 33. (a) Suppose that $\{u, v\} \in \sigma_{i} \in \Omega,\left(u^{\prime}, v^{\prime}\right) \in \mathcal{N}_{\sigma_{i}}(u, v)$ and $\sigma_{i-1}=\operatorname{Swap}_{\sigma_{i}}\left((u, v),\left(u^{\prime}, v^{\prime}\right)\right)$. Then $\hat{\psi}\left(\{\{u, v\}\}, \sigma_{i-1}\right)=\sigma_{i}$.

(b) Conversely, suppose that $\hat{\psi}\left(\{\{u, v\}\}, \sigma_{i-1}\right)=\sigma_{i}$ for $e_{i}=\{u, v\} \in E$ and $\sigma_{i-1} \in \Omega$. Then there exists unique $\left(u^{\prime}, v^{\prime}\right) \in \mathcal{N}_{\sigma_{i}}(u, v)$ such that $\sigma_{i-1}=\operatorname{Swap}_{\sigma_{i}}\left((u, v),\left(u^{\prime}, v^{\prime}\right)\right)$. Furthermore, it satisfies $\left\{u^{\prime}, v^{\prime}\right\} \notin \sigma_{i-1}-\left\{e_{i}\right\}$.

Using this lemma, we can now show establish correctness of the sampling procedure. We say that two executions of Algorithm 3 are distinct if they made different choices in line 3 for some $i \in[k]$.

Proposition 34. Algorithm 3 is well-defined, i.e. in line 3 we have $e_{i} \in \sigma_{i}$. It can generate object $\sigma_{0} \in \Omega$ if and only if $\hat{\psi}\left(M, \sigma_{0}\right)=\sigma_{k}$. Finally, distinct executions produce distinct outputs. 
Proof. The proof will have two parts corresponding to two directions.

(a) Let $\sigma_{k}, \sigma_{k-1}, \ldots$ be the sequence of objects produced by the algorithm. We will show using induction on $i=k, \ldots, 1,0$ that $\left\{e_{1}, \ldots, e_{i}\right\} \subseteq \sigma_{i}$ (and therefore line 3 for index $i$ is well-defined) and $\hat{\psi}\left(\left\{e_{i+1}, \ldots, e_{k}\right\}, \sigma_{i}\right)=\sigma_{k}$. The base case $i=k$ is trivial. Suppose the claim holds for $i \in[k]$, let us show it for $i-1$. We have $\left\{e_{1}, \ldots, e_{i}\right\} \subseteq \sigma_{i}$ by the induction hypothesis; inspecting the rule for choosing $\left(u^{\prime}, v^{\prime}\right)$, we conclude that $\left\{e_{1}, \ldots, e_{i-1}\right\} \subseteq \sigma_{i-1}$. For the second claim we can write

$$
\hat{\psi}\left(\left\{e_{i}, \ldots, e_{k}\right\}, \sigma_{i-1}\right)=\hat{\psi}\left(\left\{e_{i+1}, \ldots, e_{k}\right\}, \hat{\psi}\left(\left\{e_{i}\right\}, \sigma_{i-1}\right)\right)=\hat{\psi}\left(\left\{e_{i+1}, \ldots, e_{k}\right\}, \sigma_{i}\right)=\sigma_{k}
$$

where the first equality is by the definition of $\hat{\psi}$, the second is by Lemma 33 (a) and third is by the induction hypothesis. This concludes the argument.

(b) Suppose that $\hat{\psi}\left(M, \sigma_{0}\right)=\sigma_{k}$ for $\sigma_{0} \in \Omega$ and $M=\left\{e_{1}, \ldots, e_{k}\right\}$. Define objects $\sigma_{1}, \ldots, \sigma_{k}$ as in (14). We claim that Algorithm 3 can replicate this sequence (in the reverse order). Indeed, by Lemma 33(b) it suffices to show that for any $\left(u^{\prime}, v^{\prime}\right) \in \mathcal{N}_{\sigma_{i}}(u, v)$ with $\left\{u^{\prime}, v^{\prime}\right\} \notin \sigma_{i-1}-\left\{e_{i}\right\}$ we also have $\left(u^{\prime}, v^{\prime}\right) \in \mathcal{N}_{\sigma_{i}}(u, v)-\overrightarrow{\left\{e_{1}, \ldots, e_{i-1}\right\}}$. Suppose not, then $\left(u^{\prime}, v^{\prime}\right) \in \overrightarrow{\left\{e_{1}, \ldots, e_{i-1}\right\}}$. By Proposition 32(a) we have $\left\{e_{1}, \ldots, e_{i-1}\right\} \subseteq \sigma_{i-1}$, and so $\left\{u^{\prime}, v^{\prime}\right\} \in \sigma_{i-1}$. Thus, $\left\{u^{\prime}, v^{\prime}\right\}=e_{i}$. But $e_{i}$ does not appear in $\left\{e_{1}, \ldots, e_{i-1}\right\}$, and so we cannot have $\left(u^{\prime}, v^{\prime}\right) \in \overrightarrow{\left\{e_{1}, \ldots, e_{i-1}\right\}}$ - a contradiction.

Let us now prove that the input $M, \sigma_{k}$ and the output $\sigma_{0}$ uniquely determine choices made during the execution (this will give the last claim of the lemma). Let $\tilde{\sigma}_{k}, \ldots, \tilde{\sigma}_{1}, \tilde{\sigma}_{0}$ be the objects produced during the execution, with $\tilde{\sigma}_{k}=\sigma_{k}$ and $\tilde{\sigma}_{0}=\sigma_{0}$. Set $i=1$. By Lemma 33(a) we have $\hat{\psi}\left(\left\{e_{i}\right\}, \sigma_{i-1}\right)=\tilde{\sigma}_{i}$, implying that $\tilde{\sigma}_{i}=\sigma_{i}$ is determined uniquely. By Lemma $33(\mathrm{~b})$ the choice of $\left(u^{\prime}, v^{\prime}\right)$ in line 3 for index $i$ is also determined uniquely from $\sigma_{i-1}, \sigma_{i}$ and $e_{i}$. Repeating this argument for $i=2, \ldots, k$ (i.e. using induction) yields the claim.

We have proved that the output of Algorithm 3 is a distribution whose support is $A\left(f_{M}, \sigma_{k}\right)$. To show that this distribution is uniform, we need to observe additionally that the number of choices in line 3 for index $i$ depends on $i$ but not on the past execution history (which can be easily checked for cases [P1] and [P2]). The cardinality of $A\left(f_{M}, \sigma_{k}\right)$ is the product of these numbers over $i \in[k]$, and thus depends only on the flaw $f_{M}$ (more precisely, on $|M|$ ).

To summarize, we have constructed an atomic weakly commutative multigraph $D$, proved that Algorithm 3 samples uniformly from $A\left(f_{M}, \sigma_{k}\right)$, and the size of latter set depends only on $f_{M}$ (the latter implies strong commutativity). It can now be verified that the sampling procedure coincides with the procedure in [10] for perfect matchings in a complete graph (in the case [P1]), and with the procedure in [9, 10] for permutations (in the case [P2]).

\subsection{Application: rainbow matchings in complete graphs}

We refer to [9, 1, 10] for applications of resampling oracles for permutations and perfect matchings. Here we revisit just one application, namely a rainbow matching problem. Our primary goal is to demonstrate how the choice of the distribution $\omega^{\text {init }}$ affects the bound on the expected runtime, and also compare it with the parallel version.

Let $G=(V, E)$ be a complete graph on $2 n$ vertices such that each edge is assigned a color, and each color appears in at most $q$ edges. A perfect matching in $G$ is called rainbow if its edges have distinct colors. Achlioptas and Iliopoulos [1] showed that a rainbow matching exists if $q \leq \gamma n$ for some constant $\gamma<\frac{1}{2 e} \simeq 0.184$. Instead of $(2 \mathrm{~b})$, they used a stronger condition (3). Harvey and Vondrák [10] improved the constant to $\gamma=0.21$ by exploiting a condition with the cluster expansion correction analogous to (2). Below we redo their calculations.

Let $F$ be the set of flaws $f_{M}$ such $M$ contains two vertex-disjoint edges of the same color, and assume that we use the multigraph and relation $\sim$ constructed in the previous section. 
Proposition 35. If $\gamma=0.21$ then condition (2) can be satisfied by setting $\mu_{f}=\mu=\frac{3}{4 n^{2}}$ for $f \in F$ (if $n$ is sufficiently large).

Proof. Consider flaw $f_{M}$ where $M=\left\{\left\{v_{1}, v_{2}\right\},\left\{v_{3}, v_{4}\right\}\right\} \in F$. For node $v \in V$ let $\Gamma(v) \subseteq F$ be the set of flaws $f_{M^{\prime}}$ such that at least one of the edges in $M^{\prime}$ is incident to $v$. We have $|\Gamma(v)| \leq(2 n-1)(q-1)$ (there are at most $2 n-1$ choices for the node $v^{\prime} \in V$ matched to $v$, and then at most $q-1$ choices for the second edge of the same color as $\left.\left\{v, v^{\prime}\right\}\right)$. It can be seen that $\Gamma\left(f_{M}\right) \subseteq \Gamma\left(v_{1}\right) \cup \Gamma\left(v_{2}\right) \cup \Gamma\left(v_{3}\right) \cup \Gamma\left(v_{4}\right)$. Furthermore, any independent subset $S \in \operatorname{Ind}\left(\Gamma\left(f_{M}\right)\right)$ can be formed by selecting at most one flaw from each of $\Gamma\left(v_{i}\right)$ for $i \in[4]$. Therefore,

$$
\sum_{S \in \operatorname{Ind}\left(\Gamma\left(f_{M}\right)\right)} \mu(S) \leq \prod_{i=1}^{4}(\mu^{0}+\underbrace{\mu^{1}+\ldots+\mu^{1}}_{\left|\Gamma\left(v_{i}\right)\right| \text { times }}) \leq(1+(2 n-1)(q-1) \mu)^{4}
$$

By inspecting Algorithm 3 we can conclude that $A_{f}=(2 n-3)(2 n-1)$ for each $f \in F$. Thus, we get the following condition: there must exist $\mu>0$ such that expression

$$
\theta=\frac{1}{(2 n-3)(2 n-1) \mu} \cdot(1+(2 n-1)(q-1) \mu)^{4}
$$

is a constant smaller than 1 . Denote $\beta=(2 n-3)(2 n-1) \mu$, then

$$
\theta \leq \frac{1}{\beta} \cdot\left(1+2 n \cdot(\gamma n) \cdot \frac{\beta}{4 n^{2}} \cdot(1+o(1))\right)^{4}=\frac{\left(1+\frac{1}{2} \gamma \beta+o(1)\right)^{4}}{\beta}
$$

The last expression will be smaller than 1 (for a sufficiently large $n$ ) if $\beta=3$ and $\gamma=0.21$, where we used the constants from [10].

Let us now estimate the expression in (5). We have $|\Omega|=(2 n-1) ! !$ and $\log |\Omega|=\Theta(n \log n)$. When $\operatorname{supp}\left(\omega^{\text {init }}\right)=\left\{\sigma^{\text {init }}\right\}$ for some $\sigma^{\text {init }} \in \Omega$, we get $\gamma^{\text {init }}=|\Omega|$ and thus $T=\Omega(n \log n)$. If, on the other hand, $\omega^{\text {init }}=\omega$ then we can write

$$
\sum_{R \in \bigcup_{\sigma \in \Omega} \operatorname{Ind}\left(F_{\sigma}\right)} \mu(R) \leq \sum_{R \subseteq F} \mu(R)=\prod_{f \in F}\left(1+\mu_{f}\right)=(1+\mu)^{|F|}
$$

Observing that $|F| \leq(2 n)^{2} q=O\left(n^{3}\right)$ and $\mu=O\left(1 / n^{2}\right)$, we obtain that $T=O(|F| \log (1+\mu))=$ $O\left(n^{3} \log \left(1+O\left(\frac{1}{n^{2}}\right)\right)\right)=O\left(n^{3} \cdot \frac{1}{n^{2}}\right)=O(n)$. Thus, choosing $\omega^{\text {init }}=\omega$ leads to a better bound than initializing the algorithm with some fixed state $\sigma^{\text {init }}$. This may not be surprising, given that in the latter situation we need to consider the worst case. Note that a linear bound on the expected number of resampling steps has also been shown in [10].

We can also compute a bound on the expected number of rounds of the parallel version (Algorithm 22, assuming that $\omega^{\text {init }}=\omega$. From Theorem 8 we get

$$
T=O\left(\log \sum_{f \in F} \mu_{f}\right)=O(\log (|F| \cdot \mu))=O\left(\log \left(n^{3} \cdot \frac{1}{n^{2}}\right)\right)=O(\log n)
$$

It should be noted, however, that at the moment it is not known whether a round of Algorithm 2 can be implemented efficiently (i.e. in an expected polylogarithmic time) for matchings in a complete graph. Such implementation has only been shown for permutations [9]. We conjecture that the technique in [9] can be extended to matchings in a complete graph, but leave this question outside the scope of this work. 


\section{A Counting stable sequences: Proof of Theorems 15 and 16}

We focus on the proof of Theorem 15 , the proof of 16 will be analogous. As mentioned in Section 4.1 . there is just one essential difference between formulations of Theorem 15 and 16: the condition "there exists walk $\tau$ with $\tau \doteq W$ " is present in the definitions of both $\mathrm{Stab}_{\pi}$ and $\mathrm{Stab}_{\pi}^{\mathrm{rev}}$, and has not been "reversed". We use this condition just once, namely in the proof of Proposition 36 below, where the two cases are stated separately.

We say that a sequence $\varphi=\left(I_{1}, \ldots, I_{s}\right)$ with $s \geq 1$ is strongly stable if

(i) $I_{r} \in \operatorname{Ind}(F)$ for each $r \in[s]$,

(ii) $I_{r+1} \subseteq \Gamma\left(I_{r}\right)$ for each $r \in[s-1]$, and

(iii) $I_{r} \neq \varnothing$ for each $r \in[2, s]$.

(Compared to Definition 13, we added condition (iii), and in (ii) replaced condition $I_{r+1} \subseteq \Gamma^{+}\left(I_{r}\right)$ with a stronger condition $\left.I_{r+1} \subseteq \Gamma\left(I_{r}\right)\right)$. For a stable word $W$ let $\varphi_{W}=\left(I_{1}, \ldots, I_{s}\right)$ be the corresponding stable sequence; if $W$ is empty then $\varphi_{W}=(\varnothing)$.

Proposition 36. (a) For any $W \in \mathrm{Stab}_{\pi}$ the sequence $\varphi_{W}$ is strongly stable. (b) For any $W^{\prime} \in$ $\mathrm{Stab}_{\pi}^{\text {rev }}$ the sequence $\varphi_{W}$ is strongly stable, where $W$ is the reverse of word $W^{\prime}$.

Proof. We need to show that the sequence $\varphi_{W}=\left(I_{1}, \ldots, I_{s}\right)$ satisfies additionally property (ii). We will prove this only in the case (a), i.e. under the assumption that there exists a walk $\tau \doteq W$. The case (b), i.e. when there exists a walk $\tau \doteq W^{\prime}$, is completely analogous.

Let $W=W_{1} \ldots W_{s}$ be the partitioning of $W$ given in Definition 14. It suffices to prove that if a flaw $f$ is present in adjacent segments $W_{r}$ and $W_{r+1}$ then $f \sim f$. Suppose not: $f \nsim f$. Then by Lemma 12(b) there exists flaw $g$ between the two occurences of $f$ with $f \sim g$. We have $W_{r} W_{r+1}=\ldots f \ldots g \ldots f \ldots$. We have either $g \in W_{r}$ or $g \in W_{r+1}$, and so we must have $f \nsim g$ - a contradiction.

For a sequence $\varphi=\left(I_{1}, \ldots, I_{s}\right)$ with $s \geq 1$ let $R_{\varphi}=I_{1}$ be the first set in the sequence, and denote $|\varphi|=\sum_{r \in[s]}\left|I_{r}\right|$ and $\lambda_{\varphi}=\prod_{r \in[s]} \prod_{f \in I_{r}} \lambda_{f}$. Let Stab be the set of strongly stable sequences, $\operatorname{Stab}(R)=\left\{\varphi \in \operatorname{Stab}: R_{\varphi}=R\right\}$, and $\operatorname{Stab}(R, t)=\{\varphi \in \operatorname{Stab}(R):|\varphi| \geq t\}$.

Clearly, a $\pi$-stable word $W$ can be uniquely reconstructed from the corresponding sequence $\varphi_{W}$. Thus, $W \mapsto \varphi_{W}$ is an injective mapping from $\operatorname{Stab}_{\pi}(R, t)$ to $\operatorname{Stab}(R, t)$. Also, $\lambda_{W}=\lambda_{\varphi_{W}}$ for any $W \in \operatorname{Stab}_{\pi}(R, t)$. This means that Theorem 15 will follow from the following result.

Theorem 37. Suppose that $(\rho, \sim)$ satisfies either the cluster expansion condition (2b) or the Shearer's condition from Definition 6 . 5 Then

$$
\sum_{\varphi \in \operatorname{Stab}(R, t)} \lambda_{\varphi} \leq \mu(R) \cdot \theta^{t} \quad \forall R \in \operatorname{Ind}(F)
$$

Proof. First, assume that the Shearer's condition holds. In this case the claim has been proven in [12, 10]. To elaborate, let $p \in \mathbb{R}^{|F|}$ be the vector from Definition 6 , and define

$$
p_{\varphi}=\prod_{r \in[s]} \prod_{f \in I_{r}} p_{f}
$$

\footnotetext{
${ }^{5}$ Note that Bissacot et al. 4] proved that the cluster expansion condition implies Shearer's condition, so it would suffice to prove just the second claim. However, as mentioned in Remark 1, the definition of the cluster expansion condition was slightly stronger than condition (2b). Due to this annoying technicality we consider the two cases separately in the proof.
} 
Let $\mathrm{Stab}^{\prime}$ be the set of sequences $\phi=\left(I_{1}, \ldots, I_{s}\right)$ that satisfy conditions (i) and (ii) given in the beginning of this section. For any integer $\ell$ we can write

$$
\sum_{\substack{\varphi \in \operatorname{Stab}(R, t) \\ \text { length of } \varphi \text { is at most } \ell}} p_{\varphi} \leq \sum_{\varphi=\left(R, I_{2}, \ldots, I_{\ell}\right) \in \mathrm{Stab}^{\prime}} p_{\varphi} \leq \frac{q_{R}(p)}{q_{\varnothing}(p)} \quad \forall R \in \operatorname{Ind}(F)
$$

where the first inequality holds since for any $\varphi=\left(I_{1}, \ldots, I_{s}\right) \in \operatorname{Stab}(R, t)$ with $s \leq \ell$ there is a corresponding sequence $\varphi^{\prime}=\left(R, I_{2}, \ldots, I_{s}, \varnothing, \ldots, \varnothing\right) \in \mathrm{Stab}^{\prime}$ of length $\ell$, and the second inequality appears implicitly eq. (2) in [12] and as Lemma 5.10 in [10]. Taking a limit $\ell \rightarrow \infty$ in (19) and observing that $\mu(R)=\frac{q_{R}(p)}{q_{\varnothing}(p)}$, we get $\sum_{\varphi \in \operatorname{Stab}(R, t)} p_{\varphi} \leq \mu(R)$. From definitions we have $\lambda_{\varphi} \leq p_{\varphi} \cdot \theta^{t}$ for any $\varphi \in \operatorname{Stab}(R, t)$, this gives (17).

Now assume that condition $(2)$ holds. We say that a pair of subsets $(R, S)$ is independent if $R \cap S=\varnothing$ and $R \cup S \in \operatorname{Ind}(F)$. For such pair let $\operatorname{Stab}(R, S, t, \ell)$ be the set of sequences of the form $\varphi=\left(R, I_{2}, \ldots, I_{s}\right)$ with $s \leq \ell,|\varphi| \geq t$ that satisfy one of the following:

- $|s|=1$ (i.e. $\varphi=(R)$ ) and $S=\varnothing$;

- $|s| \geq 2, S=I_{2}-\Gamma(R)$ and $\left(I_{2}, \ldots, I_{s}\right) \in \mathrm{Stab}$.

It can be seen that $\operatorname{Stab}(R, \varnothing, t, \infty)=\operatorname{Stab}(R, t)$ for an independent set $R$.

We say that a tuple $(R, S, t, \ell)$ is valid if $(R, S)$ is an independent pair and $t \geq 0, \ell \geq 1$ are integers. We will prove the following for any valid tuple $(R, S, t, \ell)$ :

$$
\sum_{\varphi \in \operatorname{Stab}(R, S, t, \ell)} \lambda_{\varphi} \leq \mu(R) \mu(S) \cdot \theta^{t}
$$

Let us introduce a partial order $\sqsubseteq$ on tuples $(R, S, t, \ell)$ as the lexicographical order on vectors $(\ell,|R|)$ (the first component is more significant). We use induction on this partial order. The base case is given by a tuple $(R, S, t, 1)$. We can assume that $S=\varnothing$ and $t \leq|R|$ (otherwise $\operatorname{Stab}(R, S, t, 1)$ is empty). In this case $\operatorname{Stab}(R, S, t, 1)$ contains a single sequence $\varphi=(R)$, and so

$$
\sum_{\varphi \in \operatorname{Stab}(R, \varnothing, t, 1)} \lambda_{\varphi}=\prod_{f \in R} \lambda_{f} \leq \prod_{f \in R}\left(\mu_{f} \cdot \theta\right)=\mu(R) \cdot \theta^{|R|} \leq \mu(R) \cdot \theta^{t}
$$

where we used inequality $\lambda_{f} \leq \mu_{f} \cdot \theta$ that follows from condition $2 \mathrm{~b}$ with $S=\varnothing$.

Now consider a valid tuple $(R, S, t, \ell)$ with $\ell \geq 2$, and assume that the claim holds for lower tuples. Two cases are possible.

- $R=\varnothing$. We have a natural isomorphism between sets $\operatorname{Stab}(\varnothing, S, t, \ell)$ and $\operatorname{Stab}(S, \varnothing, t, \ell-1)$, namely $\operatorname{Stab}(\varnothing, S, t, \ell)=\left\{\left(\varnothing, I_{1}, \ldots, I_{r}\right) \mid\left(I_{1}, \ldots, I_{r}\right) \in \operatorname{Stab}(S, \varnothing, t, \ell-1)\right\}$. This gives

$$
\sum_{\varphi \in \operatorname{Stab}(\varnothing, S, t, \ell)} \lambda_{\varphi}=\sum_{\varphi \in \operatorname{Stab}(S, \varnothing, t, \ell-1)} \lambda_{\varphi} \leq \mu(S) \cdot \theta^{t}
$$

where in the last inequality we used the induction hypothesis.

- $R \neq \varnothing$. Pick $f \in R$, and denote $R^{-}=R-\{f\}$. Let $\tilde{\Gamma}(f)$ be the set of flaws $g \in \Gamma(f)$ that (i) can occur in the second set of a sequence $\varphi \in \operatorname{Stab}(R, S, t, \ell)$, and (ii) do belong to $\Gamma\left(f^{\prime}\right)$ 
for any $f^{\prime} \in R^{-}$. Formally, $\tilde{\Gamma}(f)=\left\{g \in \Gamma(f) \mid g \nsim f^{\prime}\right.$ for all $\left.f^{\prime} \in R \cup S\right\}$. We can write

$$
\begin{aligned}
\sum_{\varphi \in \operatorname{Stab}(R, S, t, \ell)} \lambda_{\varphi} & =\sum_{T \in \operatorname{Ind}(\tilde{\Gamma}(f))} \sum_{\varphi \in \operatorname{Stab}\left(R^{-}, S \cup T, t-1, \ell\right)} \lambda_{f} \cdot \lambda_{\varphi} \\
& \stackrel{(\text { a) }}{\leq} \lambda_{f} \sum_{T \in \operatorname{Ind}(\tilde{\Gamma}(f))} \mu\left(R^{-}\right) \mu(S \cup T) \cdot \theta^{t-1} \\
& \stackrel{\text { (b) }}{=} \mu(R) \mu(S) \theta^{t-1} \cdot \frac{\lambda_{f}}{\mu_{f}} \sum_{T \in \operatorname{Ind}(\tilde{\Gamma}(f))} \mu(T) \\
& \stackrel{\text { (c) }}{\leq} \mu(R) \mu(S) \theta^{t-1} \cdot \theta
\end{aligned}
$$

where (a) is by the induction hypothesis, (b) is true since $\mu\left(R^{-}\right)=\mu(R) / \mu_{f}$ and $\mu(S \cup T)=$ $\mu(S) \mu(T)$, and (c) follows from condition (2b).

\section{Acknowledgments}

The author thanks Dimitris Achlioptas and anonymous reviewers for useful suggestions that helped to improve the presentation of the paper. This work was supported by the European Research Council under the European Unions Seventh Framework Programme (FP7/2007-2013)/ERC grant agreement no 616160 .

\section{References}

[1] Dimitris Achlioptas and Fotis Iliopoulos. Random walks that find perfect objects and the Lovász local lemma. In 55th IEEE Annual Symposium on Foundations of Computer Science (FOCS), pages 494-503, 2014.

[2] Dimitris Achlioptas and Fotis Iliopoulos. Focused stochastic local search and the Lovász local lemma. In $S O D A, 2016$.

[3] Dimitris Achlioptas, Fotis Iliopoulos, and Vladimir Kolmogorov. Focused stochastic local search and the Lovász local lemma. arXiv, September 2018.

[4] R. Bissacot, R. Fernández, A. Procacci, and B. Scoppola. An improvement of the Lovász local lemma via cluster expansion. Combin. Probab. Comput., 20:709-719, 2011.

[5] Julia Böttcher, Yoshiharu Kohayakawa, and Aldo Procacci. Properly coloured copies and rainbow copies of large graphs with small maximum degree. Random Structures and Algorithms, 40(4):425-436, 2012.

[6] P. Erdős and L. Lovász. Problems and results on 3-chromatic hypergraphs and some related questions. In Colloq. Math. Soc. J. Bolyai, Infinite and Finite Sets, volume 10, pages 609-627. North-Holland, 1975.

[7] P. Erdős and J. Spencer. The lopsided Lovász local lemma and latin transversals. Discrete Applied Mathematics, 30:151-154, 1991. 
[8] Ioannis Giotis, Lefteris Kirousis, Kostas I. Psaromiligkos, and Dimitrios M. Thilikos. On the algorithmic Lovász local lemma and acyclic edge coloring. In Proceedings of ANALCO, 2015.

[9] David G. Harris and Aravind Srinivasan. A constructive algorithm for the Lovász local lemma on permutations. In SODA, pages 907-925, 2014.

[10] Nicholas Harvey and Jan Vondrák. An algorithmic proof of the Lovász local lemma via resampling oracles. In FOCS, 2015.

[11] Donald Knuth. The art of computer programming, Volume 4B (draft, pre-fascicle 6a). http://web.archive.org/web/20150815030301/http://www-cs-faculty.stanford. edu/ uno/news.html, July 2015.

[12] Kashyap Babu Rao Kolipaka and Mario Szegedy. Moser and Tardos meet Lovász. In Proceedings of the Forty-third Annual ACM Symposium on Theory of Computing, STOC, pages 235-244, 2011.

[13] Vladimir Kolmogorov. Commutativity in the random walk formulation of the Lovász local lemma. CoRR, abs/1506.08547v1, June 2015.

[14] Vladimir Kolmogorov. Commutativity in the algorithmic Lovász local lemma. In FOCS, 2016.

[15] Lincoln Lu, Austin Mohr, and László Székely. Quest for negative dependency graphs. In D. Bilyk, L. De Carli, A. Petukhov, A.M. Stokolos, and B.D. Wick, editors, Recent Advances in Harmonic Analysis and Applications. Springer, 2013.

[16] Austin Mohr. Applications of the lopsided Lovász local lemma regarding hypergraphs. PhD thesis, University of South Carolina, 2013.

[17] Robin A. Moser and Gábor Tardos. A constructive proof of the general Lovász local lemma. J. ACM, 57(2), 2010.

[18] Sokol Ndreca, Aldo Procacci, and Benedetto Scoppola. Improved bounds on coloring of graphs. European Journal of Combinatorics, 33(4):592-609, 2012.

[19] Wesley Pegden. An extension of the Moser-Tardos algorithmic local lemma. SIAM Journal on Discrete Mathematics, 28(2):911-917, 2014.

[20] James B. Shearer. On a problem of Spencer. Combinatorica, 5(3):241-245, 1985.

[21] Mario Szegedy. The Lovász local lemma - a survey. In Andrei A. Bulatov and Arseny M. Shur, editors, Computer Science Theory and Applications, volume 7913, pages 1-11. Springer, Lecture Notes in Computer Science, 2013. 